\title{
Hybrid Rootstocks for Valencia Sweet Orange in Rainfed Cultivation Under Tropical Savannah Climate
}

\author{
Danilo Pereira Costa $^{1}$, Eduardo Sanches Stuchi ${ }^{1,2}$, Eduardo Augusto Girardi ${ }^{1}$, Abelmon da Silva Gesteira ${ }^{1}$, \\ Maurício Antonio Coelho Filho ${ }^{1}$, Carlos Alberto da Silva Ledo ${ }^{1}$, André Luiz Fadel ${ }^{3}$, \\ André Luiz Vanucci da Silva ${ }^{4}$, Helton Carlos de Leão ${ }^{5}$, Yuri Caires Ramos ${ }^{6}$, \\ Orlando Sampaio Passos ${ }^{1} \&$ Walter dos Santos Soares Filho ${ }^{1}$ \\ ${ }^{1}$ Embrapa Mandioca e Fruticultura, Cruz das Almas, BA, Brazil \\ ${ }^{2}$ Fundação Coopercitrus Credicitrus, Bebedouro, SP, Brazil \\ ${ }^{3}$ Universidade de São Paulo, Escola Superior de Agricultura Luiz de Queiroz, Piracicaba, SP, Brazil \\ ${ }^{4}$ Rua João Pessoa, 533, Centro, Matão, SP, Brazil \\ ${ }^{5}$ Rua José Marques Pinheiro Filho, 191, Jardim Maracanã, Araraquara, SP, Brazil \\ ${ }^{6}$ Universidade Federal do Recôncavo da Bahia, Cruz das Almas, BA, Brazil \\ Correspondence: Eduardo Sanches Stuchi, Fundação Coopercitrus Credicitrus (FCC), Bebedouro, SP, Brazil. Tel: \\ 55-17-99145-1957. E-mail: eduardo.stuchi@embrapa.br
}

Received: June 22, 2020

doi:10.5539/jas.v12n11p40
Accepted: September 15, 2020

Online Published: October 15, 2020

\begin{abstract}
The performance of Valencia sweet orange grafted onto 41 hybrid citrus rootstocks was evaluated for 11 years in rainfed cultivation under tropical savannah climate (Aw type) in Brazil, in addition to three selections of the standard drought-tolerant Rangpur lime and two selections of Sunki mandarin. Drought tolerance, assessed by visual score of leaf wilting, was directly related to the mean fruit yield. Indio and Riverside citrandarins, Tropical Sunki mandarin and the hybrid TSKC $\times$ CTSW-028 were grouped with the most productive selections of Rangpur lime, all of them inducing large tree size, intermediate fruit production efficiency, and high drought tolerance. The hybrid TSK $\times$ TR English-CO was similar except by inducing a higher mean soluble solids concentration in the orange juice. A third group of rootstocks induced high yield and drought tolerance, and a mean $30 \%$ reduction in tree size that led to high production efficiency, which comprised the hybrids HTR-053, TSKC $\times($ LCR $\times$ TR)-017 and-059, TSKC $\times$ CTSW-041, LCR $\times$ TR-001 and San Diego citrandarin. The tree mortality on Rangpur lime selections was as least as $46 \%$, while more than $80 \%$ of trees grafted onto the aforementioned rootstocks survived without visual symptoms of citrus sudden death disease or graft incompatibility. The selected hybrids and Tropical Sunki mandarin also induced fruit quality, mainly soluble solids, superior to the Rangpur lime and, therefore, are potential rootstocks for rainfed cultivation of Valencia sweet orange.
\end{abstract}

Keywords: Citrus spp., Poncirus trifoliata, fruit quality, performance, fruit yield, drought tolerance

\section{Introduction}

Selection of citrus rootstocks is a challenging process because several traits must be observed for a long period, such as fruit yield, soil adaptation and reaction to pests and diseases (Castle, 2010). In Brazil and in other tropical citrus producing regions, rainfed cultivation prevails and, therefore, rootstocks should also preferably withstand seasonal water deficiency, which is aggravated by the ongoing climate change (Cimen \& Yesiloglu, 2016; Ribeiro, Espinoza-Núñes, Pompeu Junior, Mourão Filho, \& Machado, 2014; Carr, 2012). Another important issue is attending to a higher fruit quality to process NFC (not from concentrate) juice, more valued in the international market (Spreen, Gao, Fernandes Jr, \& Zansler, 2020).

Although the tristeza and drought-tolerant Rangpur lime (Citrus $\times$ limonia Osbeck) has been the main rootstock in Brazil since the 1950s, it induces poor fruit quality to the scion variety, and it is intolerant to blight, exocortis, Phythophthora spp. gummosis and citrus sudden death (CSD) (Pompeu Junior, 2005). CSD occurs in the north of Sao Paulo and in the west of Minas Gerais states (Bassanezi et al., 2003), important tropical citrus regions 
(Fundecitrus, 2019). As a result, diversification with CSD-tolerant rootstocks has increased since the 2000s (Carvalho, Girardi, Mourão Filho, Ferrarezi, \& Coletta Filho, 2019), mainly with Swingle citrumelo [C. $\times$ paradisi Macfad. $\times$ Poncirus trifoliata (L.) Raf.], Sunki [C. sunki (Hayata) hort. ex Tanaka] and Cleopatra $(C$. reshni hort. ex Tanaka) mandarins, and trifoliate orange (P. trifoliata). However, all these rootstock varieties are more sensitive to water deficit than the Rangpur lime (Pompeu Junior, 2005), increasing the rainfed cultivation risk that was only partially addressed by irrigation (Fundecitrus, 2018).

Therefore, citrus breeding programs have been creating and introducing hybrid rootstocks that must tolerate CSD either drought, besides being productive and inducing high quality fruits for NFC juice (Schinor, Cristofani-Yaly, Bastianel, \& Machado, 2013; Ramos et al., 2015). Citrandarins are hybrids of mandarins with trifoliate orange that were reported as promising rootstocks in several countries and specifically in Sao Paulo (Bowman \& Joubert, 2020; Pompeu Junior \& Blumer, 2011, 2009; Blumer \& Pompeu Junior, 2005; Pompeu Junior, Laranjeira, \& Blumer, 2002). In this work, we evaluated the long term performance of Valencia sweet orange grafted onto 41 hybrid rootstocks in rainfed cultivation under tropical savannah climate in Brazil, in addition to three selections of the standard Rangpur lime and two selections of Sunki mandarin.

\section{Material and Methods}

\subsection{Experimental Conditions}

The experiment was carried out in the municipality of Colombia, in the north of the State of Sao Paulo, Brazil $\left(20^{\circ} 1922^{\prime} \mathrm{S}, 48^{\circ} 4110^{\prime} \mathrm{W} ; 492\right.$ m.a.s.l.). The local climate is Aw, that is, tropical savannah climate like (Köppen \& Geiger, 1936), with annual mean air temperature of $26.3{ }^{\circ} \mathrm{C}$ and annual mean rainfall of $1,322 \mathrm{~mm}$ during the assessment period (Figure 1). The soil in the experimental area is a typical dark-red oxisol with a moderate A layer, mean to clayey texture, and presenting the following chemical characteristics at $0-20 \mathrm{~cm}$ depth in 2018: $\mathrm{pH}\left(\mathrm{CaCl}_{2}\right)=4.7 ; \mathrm{CEC}=51 ; \mathrm{Ca}=14 ; \mathrm{Mg}=7 ; \mathrm{K}=2.1 ; \mathrm{H}+\mathrm{Al}=28 \mathrm{mmol}_{\mathrm{c}} \mathrm{dm}^{-3}, \mathrm{~V}=45 \% ; \mathrm{P}=125 \mathrm{mg} \mathrm{dm}^{-3}$; and O. M. $=14 \mathrm{~g} \mathrm{dm}^{-3}$. 


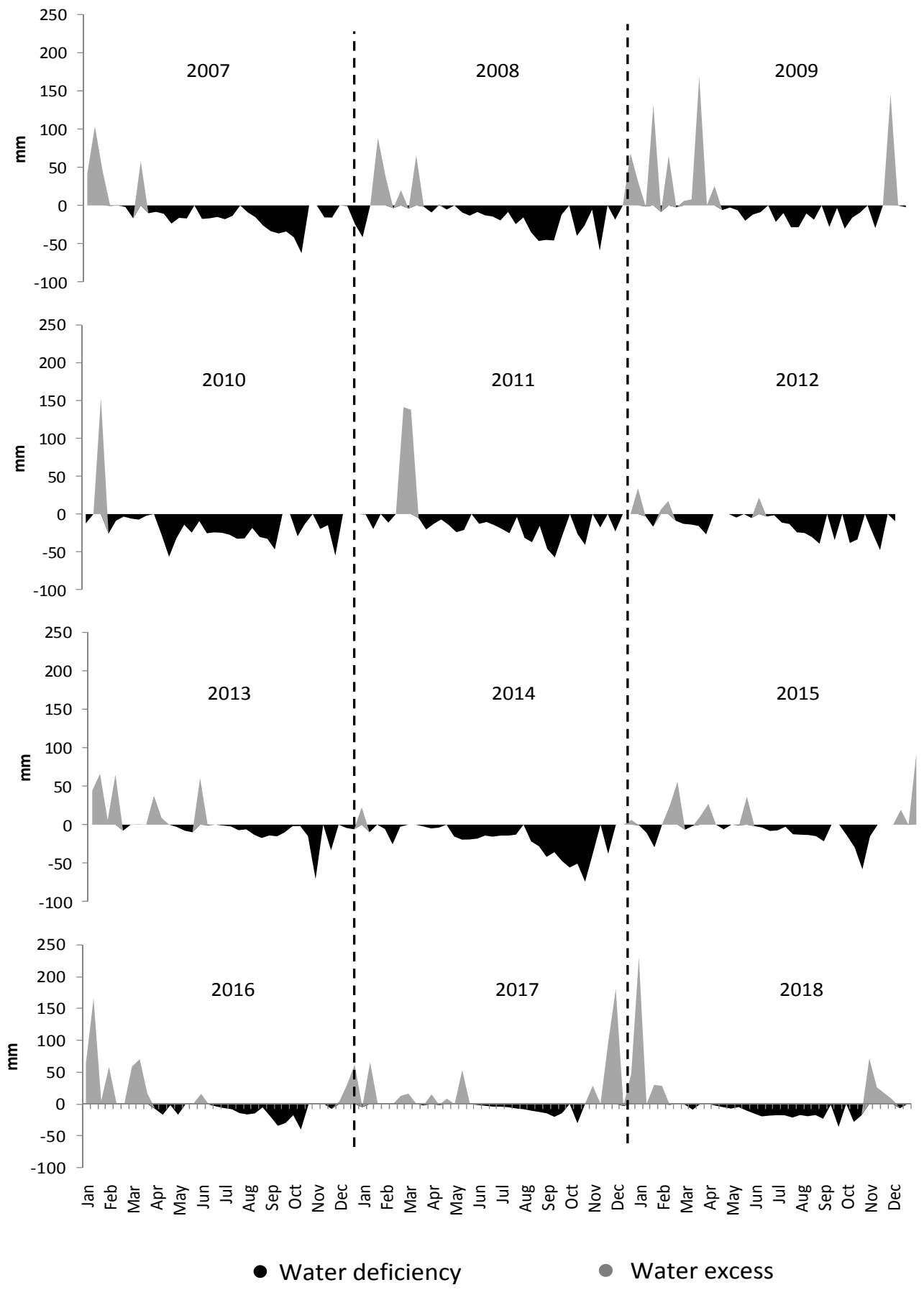

Figure 1. Water balance for the period 2007-2018 at the experimental area in Colombia, SP, Brazil, which was calculated by the Thornthwaite \& Mather method (Rolim, Sentelhas, \& Barbieri, 1998) considering $100 \mathrm{~mm}$ of water holding capacity

The planting was in 2007 at tree spacing of $6.0 \mathrm{~m}$ between-rows and $2.5 \mathrm{~m}$ in-rows (667 trees ha $\mathrm{h}^{-1}$ ), and irrigation was not used. Trees were not pruned and the incidence of huanglongbing (HLB) was neglectable. Fertilization comprised an annual mean rate of $320 \mathrm{~g} \mathrm{~N}, 130 \mathrm{~g} \mathrm{P}_{2} \mathrm{O}_{5}$ and $230 \mathrm{~g} \mathrm{~K}_{2} \mathrm{O}$ per tree from 2012 to 2018 . Limestone was applied annually at $2.2 \mathrm{t} \mathrm{ha}^{-1}$, and other cultural practices were usual to the citrus industry in Brazil (Passos et al., 2018).

\subsection{Plant Material and Experimental Design}

Valencia IAC sweet orange $[C . \times$ sinensis $(\mathrm{L}$.) Osbeck] was used as the scion variety. Forty-one hybrid citrus rootstocks created or introduced by the Citrus Breeding Program of Embrapa Mandioca e Fruticultura were 
evaluated, in addition to three selections of Rangpur lime (with Santa Cruz as the control) and two selections of Sunki mandarin (Table 1). The experimental design was in randomized blocks, with 46 treatments (rootstocks), three replications and five trees in the plot, given a total of 690 trees.

Table 1. Cultivar name or hybrid acronym, and species or parental of 46 citrus rootstocks grafted with Valencia IAC sweet orange [Citrus $\times$ sinensis (L.) Osbeck]

\begin{tabular}{|c|c|}
\hline Cultivar name or hybrid acronym ${ }^{1}$ & Species or parental \\
\hline 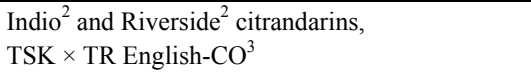 & Sunki mandarin [C. sunki (Hayata) hort. ex Tanaka] $\times$ Poncirus trifoliata (L.) Raf. cv. English \\
\hline San Diego ${ }^{2}$ citrandarin & Sunki mandarin $\times P$. trifoliata $\mathrm{cv}$. Swingle \\
\hline Santa Cruz, CNPMF ${ }^{4}-003$ and-004 & Rangpur lime $(C . \times$ limonia Osbeck $)$ \\
\hline TSKC $\times$ CTSW-019, 028, 033, 041 and 064 & Common Sunki mandarin $(C$. sunki $) \times$ Swingle citrumelo $(C . \times$ paradisi Macfad. $\times$ P. trifoliata $)$ \\
\hline Maravilha and Tropical selections & Sunki mandarin (C. sunki) \\
\hline HTR-051, 053, 069 and 116 & Hybrids involving $P$. trifoliata \\
\hline TSKC $\times(\mathrm{LCR} \times \mathrm{TR})-001,017,018,059$ and 073 & Common Sunki mandarin $\times[C . \times$ limonia $\times P$. trifoliata $]$ \\
\hline $\mathrm{LRF} \times(\mathrm{LCR} \times \mathrm{TR})-005$ & Florida rough lemon $(C . \times$ jambhiri Lush. $) \times[C . \times$ limonia $\times P$. trifoliata $]$ \\
\hline $\mathrm{LCR} \times \mathrm{TR}-001$ & C. $\times$ limonia $\times$ P. trifoliata \\
\hline LVK $\times$ LCR-010 and 038 & Volkamer lemon $[C . \times$ volkameriana $($ Risso) V. Ten. \& Pasq. $] \times$ Rangpur lime \\
\hline TSK $\times$ TR Benecke-CO & Sunki mandarin $\times P$. trifoliata $\mathrm{cv}$. Benecke \\
\hline TSKC $\times$ CTTR-002 & Sunki mandarin $\times$ Troyer citrange $(C . \times$ sinensis $\times P$. trifoliata $)$ \\
\hline CLEO $\times$ CTCZ-226 & Cleopatra mandarin $($ C. reshni $i$ hort. ex Tanaka $) \times$ Carrizo citrange \\
\hline LVK $\times$ LVA-009 & C. $\times$ volkameriana $\times$ Valencia sweet orange $(C . \times$ sinensis $)$ \\
\hline TSKC $\times$ CTQT 1434-010 & $\begin{array}{l}\text { Common Sunki mandarin } \times \text { Thomasville citrangequat [Fortunella margarita (Lour.) Swingle } \\
\times \text { Willits citrange] cv. } 1434\end{array}$ \\
\hline TSKC $\times$ LHA-006 and 011 & Common Sunki mandarin $\times$ Hamlin sweet orange \\
\hline TSKC $\times$ CTARG-001 and 036 & Common Sunki mandarin $\times$ Argentina citrange \\
\hline TSKFL $\times$ CTC $25-010$ & Sunki mandarin Florida selection $\times \mathrm{C}-25$ citrange \\
\hline TSKFL $\times$ CTTR-008, 012 and 022 & Sunki mandarin Florida selection $\times$ Troyer citrange \\
\hline TSKC $\times$ CTQT 1439-004 and 026 & Common Sunki mandarin $\times$ Thomasville citrangequat cv. 1439 \\
\hline $\mathrm{CLEO} \times \mathrm{TR}$ Rubidoux $-\mathrm{CO}$ & Cleopatra mandarin $\times P$. trifoliata $\mathrm{cv}$. Rubidoux \\
\hline LCREEL $\times$ CTSW-001 & Rangpur lime cv. EEL $\times$ Swingle citrumelo \\
\hline TSKC $\times$ CTRK-001 & Common Sunki mandarin $\times$ Rusk citrange \\
\hline TSK $\times$ Alemow-CO & Sunki mandarin $\times$ Alemow (C. macrophylla Wester) \\
\hline
\end{tabular}

Note. ${ }^{1}$ Acronyms used by the Citrus Breeding Program of Embrapa Mandioca e Fruticultura, with the sequential numbers indicating different hybrids of the same cross. ${ }^{2}$ Introduced in Brazil in 1963 from the USDA, Indio, California. ${ }^{3}$ Palmira, Colombia, from which the accession was introduced in Brazil. ${ }^{4}$ Embrapa Mandioca e Fruticultura.

\subsection{Tree Size}

Tree height $(\mathrm{H})$ was measured from the soil level to the canopy apex, and the mean canopy diameter (D) was calculated as the average between the equatorial diameter measured perpendicularly and parallel to the row. Tree size was measured from 2009 to 2015 in March/April of each year, but only data from 2015 (full bearing age) is presented. The canopy volume $\left(\mathrm{V}, \mathrm{m}^{3}\right)$ was calculated by the expression:

$$
\mathrm{V}=2 / 3 \pi(\mathrm{D} / 2)^{2} \times \mathrm{H}
$$

Where, $\mathrm{D}=$ Diameter and $\mathrm{H}=$ Height of plant (Zekri, 2000). The rootstocks were classified by the tree size according to Castle and Phillips (1977): dwarfing, semi-dwarfing, semi-standard, standard and extra-standard rootstocks had, respectively, $<40 \%, 41-60 \%, 61-80 \%, 81-100 \%$ and $>100 \%$ of the tree size of the standard rootstock (Santa Cruz Rangpur lime).

\subsection{Fruit Yield, Production Efficiency and Alternate Bearing}

Fruits were harvested in October/November in each year, after preliminary fruit analyses by the farm manager. Fruit yield was weighed from 2009 to 2015 and expressed as $\mathrm{kg}^{-1} \mathrm{tree}^{-1}$ for the mean of the seven initial harvests. In 2016 and 2017, expressive fruit drop occurred before harvesting could be performed, hence fruit yield was not computed. In 2018, fruit yield was weighed only for some rootstocks selected for their previous performance. The production efficiency $\left(\mathrm{PE}, \mathrm{kg} \mathrm{m}^{-3}\right)$ was calculated using the expression:

$$
\mathrm{PE}=\mathrm{FY} / \mathrm{V}
$$


Where, FY = Fruts Yield and V = Canopy volume, average from 2009 to 2015 (Fadel, Stuchi, Couto, Ramos, \& Mourao Filho, 2018). The alternate bearing index (ABI) was calculated by the expression:

$$
\mathrm{ABI}=1 /(\mathrm{n}-1) \times\left\{\left|\mathrm{a}_{2}-\mathrm{a}_{1} / /\left(\mathrm{a}_{2}+\mathrm{a}_{1}\right)+\right| \mathrm{a}_{3}-\mathrm{a}_{2}\left|/\left(\mathrm{a}_{3}+\mathrm{a}_{2}\right)+\ldots+\right| \mathrm{a}_{\mathrm{n}}-\mathrm{a}_{\mathrm{n}-1} \mid /\left(\mathrm{a}_{\mathrm{n}}+\mathrm{a}_{\mathrm{n}-1}\right)\right\}
$$

Where, $n=$ the number of evaluated harvests, and $a_{1}, a_{2}, \ldots a_{n-1}, a_{n}=$ the fruit weight in the correspondent years (Pearce \& Dobersek-Urbanc, 1967).

\subsection{Tolerance to Drought}

Rootstock tolerance to drought was visually assessed based on the leaf wilting during the most severe water deficit period in each year (Figure 1). Two evaluators simultaneously attributed independent grades to each tree according to a score adapted from Fadel et al. (2018): 1) low tolerance (leaf drop, shoot dieback and general leaf wilting in the canopy); 2) intermediate tolerance (partial leaf wilting in the canopy); and 3) high tolerance (no leaf wilting in the canopy). The mean grade was calculated for the period 2010-2017 except 2015.

\subsection{Tree Survival Rate and Graft Incompatibility}

Tree survival rate was calculated by the relation between the cumulative number of dead trees until 2018 and the total number of trees planted for each plot, and expressed in percentage basis. Graft compatibility was evaluated in November 2017 only for 19 selected rootstocks, including the control, due to presenting more promising horticultural traits to that date. The trunk bark from a $3 \times 5 \mathrm{~cm}$ area was removed on the graft union with a penknife. Visual assessment was performed by two independent evaluators on six plants of each treatment, using the following score: 1) no symptoms of graft incompatibility; 2) fine line separating the scion and the rootstock tissues; 3) marked line separating the scion and the rootstock tissues; and 4) sharp and deep line separating the scion and the rootstock tissues with rootstock phloem yellowing, necrosis and gum exudation in the graft union (Fadel et al., 2019).

\subsection{Fruit Quality}

From 2009 to 2015, samples of 10 fruits were picked on all quadrants of the canopy in each plot, just before harvesting, with fruit quality variables presented as the average of this period. The fruit weight was measured on a digital scale. The juice was obtained from a point-of-sale small extractor (Otto 1800, OIC, Limeira, Brazil) and the juice content (JC) was calculated by the expression:

$$
\mathrm{JC}=(\mathrm{JW} \times 100) / \mathrm{FW}
$$

Where, $\mathrm{JW}=$ juice weight, $\mathrm{FW}=$ fruit weight, expressed as percentage basis. The concentration of total soluble solids (SS) in the juice was measured with a digital refractometer (Palette PR-101, Atago, Tokyo, Japan), and expressed as ${ }^{\circ}$ Brix. The titratable acidity (TA) was measured by the titration with sodium hydroxide $(0.3125 \mathrm{~N})$ and expressed as percentage basis. The fruit maturity indice (MI) was estimated by the maturity index by the expression:

$$
\mathrm{MI}=\mathrm{SS} / \mathrm{TA}
$$

Where, $\mathrm{SS}=$ soluble solids and $\mathrm{TA}=$ titratable acidity. The technological index (TI) was calculated by the expression:

$$
\mathrm{TI}=(\mathrm{JC} \times \mathrm{SS} \times 40.8) / 10.000
$$

Where, $\mathrm{JC}=$ juice weight, $\mathrm{SS}=$ soluble solids and $40.8 \mathrm{~kg}$ is the weight of the standard industrial box of sweet orange.

\subsection{Statistical Analyses}

Data were submitted to variance analysis and the means were grouped by the Scott-Knott test $(\mathrm{p}<0.05$ and $\mathrm{p}<$ 0.15 for ABI). Multivariate analyses were performed using variables that were selected as the most relevant for orange cropping for juice processing, according to the authors and the farm managers experience: mean fruit yield, mean fruit production efficiency, mean soluble solids concentration in the juice, mean juice content of the fruit, drought tolerance grade and plant height. A principal component analysis (PCA) was performed on the normalized data, and the auto-values (variance associated to each principal component) were estimated by the typical square-roots of the covariance, and the auto-vectors (set of weighted coefficients of the principal components) were estimated by the corresponding elements of the typical vectors, according to Cruz, Regazzi and Carneiro (2004). The hierarchical clustering of the individual and simultaneous analyses from the matrices of genetic distance was obtained by the UPGMA (Unweighted Pair Group Method with Arithmetic Mean) method (Sneath \& Sokal, 1973). The clustering validation was determined by the cofenetic correlation coefficient (CCC) according to Sokal and Rohlf (1962). The significance of the CCCs was calculated by the 
Mantels $\mathrm{t}$ test with 1,000 permutations (Mantel, 1967). The genetic distance matrices were calculated for individual analyses and CCCs, and the genetic distance matrix was based on the Gowers algorithm. The dissimilarity dendogram was calculated and the criterion to define the ideal number of groups was the pseudo-t2 (Mingotti, 2005), which was calculated with the "NbClust" package of the R program (Charrad, Ghazzali, Boiteau, \& Niknafs, 2013).

\section{Results}

\subsection{Tree Size}

The evaluated rootstocks were grouped in two and three classes of tree diameter and height, respectively, which resulted in two classes of canopy volume presenting an average of 28.9 and $18.1 \mathrm{~m}^{3}$, respectively, at nine years after planting (Table 2). According to the tree size classification of Castle and Philips (1977), 15.2, 21.7, 45.7 and $17.4 \%$ of the rootstocks were respectively classified as extra-standard, standard, semi-standard and semi-dwarfing in relation to the Santa Cruz Rangpur lime.

Table 2. Tree size (2015), fruit yield (FY, 2009-2015 and 2018) and production efficiency (PE, mean of 2009-2015) of Valencia sweet orange [Citrus $\times$ sinensis (L.) Osbeck] grafted onto 46 rootstocks in rainfed cultivation under tropical savannah climate (Aw type) in Colombia, Northern Sao Paulo State, Brazil

\begin{tabular}{|c|c|c|c|c|c|c|c|c|c|c|c|c|}
\hline \multirow{3}{*}{$\begin{array}{l}\text { Rootstock } \\
\text { Indio citrandarin }\end{array}$} & \multicolumn{2}{|c|}{ Diameter } & \multirow{2}{*}{\multicolumn{2}{|c|}{ 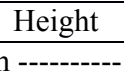 }} & \multirow{2}{*}{\multicolumn{2}{|c|}{$\begin{array}{l}\text { Canopy volume } \\
--m^{3}-\end{array}$}} & \multirow{2}{*}{\multicolumn{2}{|c|}{$\begin{array}{l}\text { Mean Yield 2009-2015 } \\
-1\end{array}$}} & \multirow{2}{*}{\multicolumn{2}{|c|}{ Yield 2018}} & \multirow{2}{*}{\multicolumn{2}{|c|}{$\frac{\mathrm{PE}}{--\mathrm{kg} \mathrm{m}^{-3}--}$}} \\
\hline & & & & & & & & & & & & \\
\hline & 3.99 & a & 3.78 & $\mathrm{a}$ & 32.50 & $\mathrm{a}$ & 45.80 & $\mathrm{a}$ & 72.00 & $\mathrm{a}$ & 3.17 & $\mathrm{a}$ \\
\hline Riverside citrandarin & 4.04 & $\mathrm{a}$ & 3.82 & a & 33.40 & $\mathrm{a}$ & 35.80 & $\mathrm{~b}$ & 76.67 & $\mathrm{a}$ & 2.48 & $\mathrm{~b}$ \\
\hline San Diego citrandarin & 3.82 & $\mathrm{a}$ & 3.35 & $\mathrm{~b}$ & 25.70 & $\mathrm{a}$ & 43.60 & $\mathrm{a}$ & 60.33 & $\mathrm{a}$ & 3.51 & $\mathrm{a}$ \\
\hline CLEO × CTCZ-226 & 3.56 & b & 3.22 & $\mathrm{~b}$ & 21.40 & $\mathrm{~b}$ & 32.90 & $\mathrm{~b}$ & - & & 3.06 & a \\
\hline CLEO $\times$ TR Rubidoux-CO & 3.10 & b & 2.72 & $\mathrm{c}$ & 13.90 & $\mathrm{~b}$ & 22.90 & $\mathrm{~d}$ & - & & 2.45 & $\mathrm{~b}$ \\
\hline HTR-051 & 3.44 & b & 3.28 & $\mathrm{~b}$ & 20.30 & $\mathrm{~b}$ & 34.90 & $\mathrm{~b}$ & 61.33 & $\mathrm{a}$ & 3.48 & a \\
\hline HTR-053 & 3.46 & b & 3.34 & $\mathrm{~b}$ & 21.00 & $\mathrm{~b}$ & 41.00 & a & 54.33 & $\mathrm{~b}$ & 3.87 & $\mathrm{a}$ \\
\hline HTR-069 & 3.18 & $\mathrm{~b}$ & 3.07 & $\mathrm{c}$ & 16.30 & $\mathrm{~b}$ & 30.70 & $\mathrm{c}$ & - & & 4.33 & a \\
\hline HTR-116 & 3.23 & $\mathrm{~b}$ & 3.06 & $\mathrm{c}$ & 16.70 & $\mathrm{~b}$ & 35.10 & $\mathrm{~b}$ & 33.33 & $\mathrm{~b}$ & 4.07 & $\mathrm{a}$ \\
\hline $\mathrm{LCR} \times \mathrm{TR}-001$ & 3.04 & $\mathrm{~b}$ & 3.00 & $\mathrm{c}$ & 14.80 & $\mathrm{~b}$ & 36.00 & $\mathrm{~b}$ & 74.33 & $\mathrm{a}$ & 4.14 & $\mathrm{a}$ \\
\hline LCREEL $\times$ CTSW-001 & 3.28 & $\mathrm{~b}$ & 3.22 & $\mathrm{~b}$ & 18.10 & $\mathrm{~b}$ & 21.30 & $\mathrm{~d}$ & - & & 2.51 & $\mathrm{~b}$ \\
\hline CNPMF-003 Rangpur lime & 3.42 & $\mathrm{~b}$ & 3.26 & $\mathrm{~b}$ & 19.90 & $\mathrm{~b}$ & 34.20 & $\mathrm{~b}$ & 39.00 & $\mathrm{~b}$ & 3.32 & $\mathrm{a}$ \\
\hline CNPMF-004 Rangpur lime & 3.84 & $\mathrm{a}$ & 3.80 & $\mathrm{a}$ & 29.40 & $\mathrm{a}$ & 43.20 & $\mathrm{a}$ & 52.00 & $\mathrm{~b}$ & 3.19 & a \\
\hline Santa Cruz Rangpur lime & 3.78 & $\mathrm{a}$ & 3.59 & $\mathrm{a}$ & 26.90 & $\mathrm{a}$ & 39.40 & $\mathrm{a}$ & 48.67 & $\mathrm{~b}$ & 3.41 & a \\
\hline $\mathrm{LRF} \times(\mathrm{LCR} \times \mathrm{TR})-005$ & 3.93 & $\mathrm{a}$ & 3.31 & $\mathrm{~b}$ & 26.70 & $\mathrm{a}$ & 37.90 & $\mathrm{~b}$ & 61.33 & $\mathrm{a}$ & 2.57 & $\mathrm{~b}$ \\
\hline LVK $\times$ LCR- 010 & 3.43 & $\mathrm{~b}$ & 3.20 & $\mathrm{~b}$ & 20.00 & $\mathrm{~b}$ & 34.60 & $\mathrm{~b}$ & - & & 3.33 & $\mathrm{a}$ \\
\hline LVK $\times$ LCR -038 & 2.92 & $\mathrm{~b}$ & 2.93 & $\mathrm{c}$ & 13.20 & $\mathrm{~b}$ & 33.90 & $\mathrm{~b}$ & 50.67 & $\mathrm{~b}$ & 4.28 & $\mathrm{a}$ \\
\hline LVK $\times$ LVA-009 & 3.55 & $\mathrm{~b}$ & 3.27 & $\mathrm{~b}$ & 22.00 & $\mathrm{~b}$ & 30.80 & $\mathrm{c}$ & - & & 2.72 & $\mathrm{~b}$ \\
\hline TSK $\times$ Alemow $-\mathrm{CO}$ & 3.18 & $\mathrm{~b}$ & 3.01 & $\mathrm{c}$ & 15.90 & $\mathrm{~b}$ & 17.80 & $\mathrm{~d}$ & - & & 2.62 & $\mathrm{~b}$ \\
\hline TSK × TR English-CO & 4.09 & $\mathrm{a}$ & 3.76 & $\mathrm{a}$ & 32.80 & $\mathrm{a}$ & 45.40 & $\mathrm{a}$ & 78.67 & $\mathrm{a}$ & 2.90 & $\mathrm{~b}$ \\
\hline Maravilha Sunki mandarin & 3.59 & $\mathrm{a}$ & 3.34 & $\mathrm{~b}$ & 23.00 & $\mathrm{~b}$ & 24.80 & $\mathrm{~d}$ & - & & 1.72 & $\mathrm{~b}$ \\
\hline Tropical Sunki mandarin & 3.64 & $\mathrm{a}$ & 3.73 & $\mathrm{a}$ & 25.90 & $\mathrm{a}$ & 41.60 & $\mathrm{a}$ & 50.67 & $\mathrm{~b}$ & 2.72 & $\mathrm{~b}$ \\
\hline TSK $\times$ CTTR-002 & 3.32 & $\mathrm{~b}$ & 3.30 & $\mathrm{~b}$ & 19.10 & $\mathrm{~b}$ & 32.40 & $\mathrm{~b}$ & - & & 2.76 & $\mathrm{~b}$ \\
\hline TSK $\times$ TR Benecke-CO & 3.34 & $\mathrm{~b}$ & 3.12 & $\mathrm{~b}$ & 18.80 & $\mathrm{~b}$ & 33.40 & $\mathrm{~b}$ & 65.33 & $\mathrm{a}$ & 2.88 & $\mathrm{~b}$ \\
\hline $\mathrm{TSKC} \times(\mathrm{LCR} \times \mathrm{TR})-001$ & 3.22 & $\mathrm{~b}$ & 2.97 & $\mathrm{c}$ & 16.20 & $\mathrm{~b}$ & 34.50 & $\mathrm{~b}$ & - & & 3.76 & a \\
\hline $\mathrm{TSKC} \times(\mathrm{LCR} \times \mathrm{TR})-017$ & 3.28 & $\mathrm{~b}$ & 3.13 & $\mathrm{~b}$ & 17.70 & $\mathrm{~b}$ & 39.40 & a & 66.67 & $\mathrm{a}$ & 4.08 & $\mathrm{a}$ \\
\hline $\mathrm{TSKC} \times(\mathrm{LCR} \times \mathrm{TR})-018$ & 3.33 & $\mathrm{~b}$ & 3.13 & $\mathrm{~b}$ & 18.30 & $\mathrm{~b}$ & 30.40 & $\mathrm{c}$ & - & & 3.31 & $\mathrm{a}$ \\
\hline $\mathrm{TSKC} \times(\mathrm{LCR} \times \mathrm{TR})-059$ & 3.39 & $\mathrm{~b}$ & 3.16 & $\mathrm{~b}$ & 19.50 & $\mathrm{~b}$ & 37.40 & $\mathrm{~b}$ & 64.67 & $\mathrm{a}$ & 4.20 & $\mathrm{a}$ \\
\hline $\mathrm{TSKC} \times(\mathrm{LCR} \times \mathrm{TR})-073$ & 3.53 & $\mathrm{~b}$ & 3.49 & $\mathrm{a}$ & 22.70 & $\mathrm{~b}$ & 36.20 & $\mathrm{~b}$ & - & & 3.32 & a \\
\hline TSKC $\times$ CTARG-001 & 4.00 & $\mathrm{a}$ & 3.98 & $\mathrm{a}$ & 33.50 & $\mathrm{a}$ & 28.40 & $\mathrm{c}$ & - & & 1.66 & $\mathrm{~b}$ \\
\hline TSKC × CTARG-036 & 3.34 & $\mathrm{~b}$ & 3.21 & $\mathrm{~b}$ & 19.20 & $\mathrm{~b}$ & 24.50 & $\mathrm{~d}$ & - & & 2.26 & $\mathrm{~b}$ \\
\hline TSKC $\times$ CTQT 1434-010 & 3.31 & $\mathrm{~b}$ & 2.93 & $\mathrm{c}$ & 16.80 & $\mathrm{~b}$ & 30.70 & $\mathrm{c}$ & - & & 3.75 & $\mathrm{a}$ \\
\hline TSKC $\times$ CTQT 1439-004 & 3.78 & $\mathrm{a}$ & 3.34 & $\mathrm{~b}$ & 24.90 & $\mathrm{a}$ & 29.70 & $\mathrm{c}$ & - & & 2.09 & $\mathrm{~b}$ \\
\hline TSKC $\times$ CTQT 1439-026 & 3.13 & $\mathrm{~b}$ & 2.87 & $\mathrm{c}$ & 14.90 & $\mathrm{~b}$ & 26.90 & $\mathrm{c}$ & - & & 3.54 & $\mathrm{a}$ \\
\hline TSKC $\times$ CTRK-001 & 3.23 & $\mathrm{~b}$ & 3.32 & $\mathrm{~b}$ & 18.10 & $\mathrm{~b}$ & 20.70 & $\mathrm{~d}$ & - & & 2.00 & $\mathrm{~b}$ \\
\hline TSKC $\times$ CTSW-019 & 3.92 & $\mathrm{a}$ & 3.73 & $\mathrm{a}$ & 30.40 & $\mathrm{a}$ & 33.60 & $\mathrm{~b}$ & - & & 2.02 & $\mathrm{~b}$ \\
\hline TSKC $\times$ CTSW -028 & 3.82 & $\mathrm{a}$ & 3.70 & $\mathrm{a}$ & 28.80 & $\mathrm{a}$ & 42.40 & $\mathrm{a}$ & 77.33 & $\mathrm{a}$ & 3.14 & $\mathrm{a}$ \\
\hline TSKC $\times$ CTSW -033 & 3.19 & $\mathrm{~b}$ & 3.07 & $\mathrm{c}$ & 16.50 & $\mathrm{~b}$ & 29.70 & $\mathrm{c}$ & - & & 3.62 & $\mathrm{a}$ \\
\hline TSKC $\times$ CTSW -041 & 3.32 & $\mathrm{~b}$ & 3.24 & $\mathrm{~b}$ & 18.90 & $\mathrm{~b}$ & 38.90 & $\mathrm{a}$ & 54.00 & $\mathrm{~b}$ & 3.77 & $\mathrm{a}$ \\
\hline
\end{tabular}




\begin{tabular}{|c|c|c|c|c|c|c|c|c|c|c|}
\hline TSKC $\times$ CTSW-064 & $3.30 \mathrm{~b}$ & 3.08 & c & 17.80 & $\mathrm{~b}$ & 29.30 & $\mathrm{c}$ & - & 3.92 & $\mathrm{a}$ \\
\hline TSKC × LHA-006 & $3.67 \mathrm{a}$ & 3.41 & $\mathrm{~b}$ & 24.30 & $\mathrm{a}$ & 30.10 & $\mathrm{c}$ & - & 2.22 & $b$ \\
\hline TSKC $\times$ LHA-011 & $3.53 \mathrm{~b}$ & 3.43 & $\mathrm{a}$ & 22.40 & $\mathrm{~b}$ & 24.60 & d & - & 1.95 & $\mathrm{~b}$ \\
\hline TSKFL $\times$ CTC $25-010$ & $3.17 \mathrm{~b}$ & 2.87 & $\mathrm{c}$ & 15.20 & $\mathrm{~b}$ & 28.20 & $\mathrm{c}$ & - & 3.76 & a \\
\hline TSKFL $\times$ CTTR-008 & $3.11 \mathrm{~b}$ & 3.04 & $\mathrm{c}$ & 15.30 & $b$ & 22.40 & d & - & 2.52 & b \\
\hline TSKFL $\times$ CTTR -012 & $3.07 \mathrm{~b}$ & 3.11 & $\mathrm{~b}$ & 15.30 & $\mathrm{~b}$ & 27.50 & $\mathrm{c}$ & - & 3.52 & a \\
\hline TSKFL $\times$ CTTR -022 & $3.35 \mathrm{~b}$ & 2.87 & $\mathrm{c}$ & 16.90 & $\mathrm{~b}$ & 23.00 & d & - & 2.88 & $\mathrm{~b}$ \\
\hline $\mathrm{P}$ & $* *$ & $* *$ & & $* *$ & & $* *$ & & $* *$ & $* *$ & \\
\hline $\mathrm{CV}(\%)$ & 8.6 & 6.32 & & 22.88 & & 16.14 & & 19.69 & 20.37 & \\
\hline Mean & 3.46 & 3.27 & & 21.10 & & 32.60 & & 60.07 & 3.10 & \\
\hline
\end{tabular}

Note. Means followed by the same letters in the column belong to the same group by the Scott-Knott test. (ns) not significant, $\left({ }^{*}\right)$ significant at $P<0.05$ and $(* *)$ significant at $P<0.01$. (-) not evaluated.

\subsection{Fruit Yield, Production Efficiency and Alternate Bearing}

The most productive trees in 2009-2015 were grafted on Indio, San Diego and TSK $\times$ TR English-CO citrandarins, Santa Cruz and CNPMF-004 Rangpur limes, TSKC $\times$ CTSW-028 and-041, Tropical Sunki mandarin, HTR-053 and TSKC $\times($ LCR $\times$ TR)-017. Conversely, CLEO $\times$ TR Rubidoux-CO and Maravilha Sunki mandarin were among the least productive. In 2018, the former rootstocks besides LCR $\times$ TR-001, TSKC $\times(\mathrm{TR} \times \mathrm{LCR})-059, \mathrm{TSK} \times \mathrm{TR}$ Benecke-CO, HTR-051, LRF $\times(\mathrm{LCR} \times \mathrm{TR})-005$ and Riverside citrandarin induced the highest fruit yield, even though all Rangpur lime selections, Tropical Sunki mandarin, HTR-053 and TSKC $\times$ CTSW-041 decreased the yield (Table 2).

The Rangpur lime selections and the majority of semi-standard and semi-dwarfing rootstocks induced higher production efficiency, with 56.5 and $43.5 \%$ of the rootstocks inducing, respectively, a mean of 3.6 and $2.4 \mathrm{~kg} \mathrm{~m}^{-3}$ to the Valencia sweet orange (Table 2). Tropical Sunki mandarin had the highest ABI, probably because it was a little late bearer, and about $67 \%$ of the evaluated rootstocks induced the lowest ABI $(0.25$ to 0.36$)$, including all selections of Rangpur lime, Indio and San Diego citrandarins, TSKC $\times$ CTSW-028 and HTR-053 that were also among the most productive ones (Table 3 ).

\subsection{Tolerance to Drought}

The hybrids LCR $\times$ TR-001, TSKC $\times($ LCR $\times$ TR $)-017$ and-059, TSK $\times$ CTTR-002, TSKC $\times$ CTSW-028 and-041, HTR-053, LVK $\times$ LCR-038, San Diego and Riverside citrandarins and Tropical Sunki mandarin were grouped alongside all selections of Rangpur lime as the most drought-tolerant rootstocks based on the visual assessment of leaf wilting during drought conditions (Table 3; Figure 1). The mean fruit yield in 2009-2015 was positively related to the mean grade of tolerance to drought $(P<0.01)$ (Figure 2).

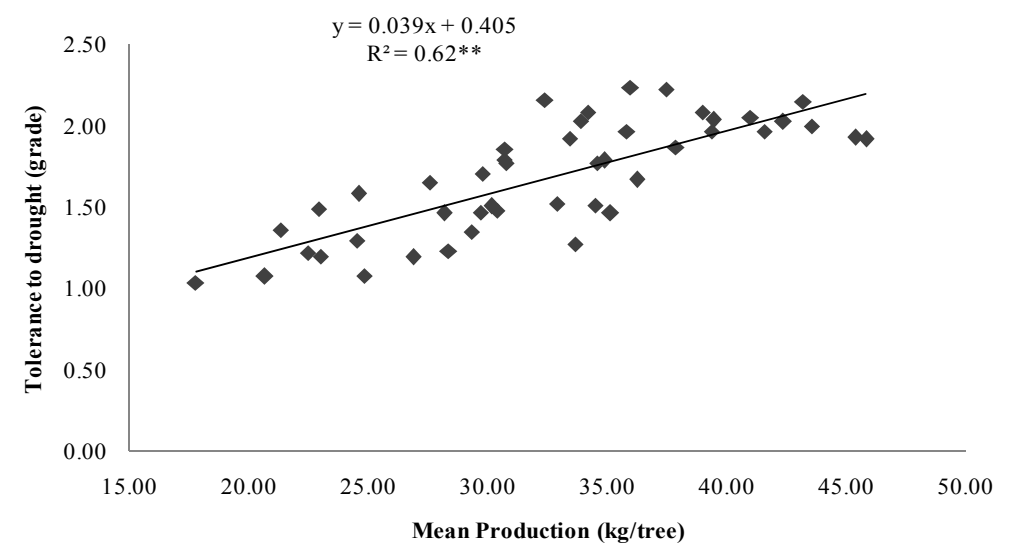

Figure 2. Linear regression between the mean grade of tolerance to drought (2010-2017, except 2015) and the mean fruit yield (2009-2015) of Valencia sweet orange grafted onto 46 rootstocks in rainfed cultivation under tropical savannah climate (Aw type) in Colombia, Northern Sao Paulo State, Brazil. Each dot corresponds to the mean values of each rootstock variety $(n=3)$. Regression model significant at $(P<0.01)$. Tolerance to drought was assessed by visual scoring as adapted from Fadel et al. (2018): 1) low tolerance (leaf drop, shoot dieback and general leaf wilting in the canopy); 2) intermediate tolerance (partial leaf wilting in the canopy); and 3) high tolerance (no leaf wilting in the canopy) 


\subsection{Tree Survival Rate and Graft Incompatibility}

Eleven years after planting, only $13.3 \%$ of plants grafted onto LVK $\times$ LVA-009 were still alive. Eight rootstocks including the Santa Cruz Rangpur lime and Maravilha Sunki mandarin resulted in a mean tree survival of 52.5\%, whereas the remaining rootstocks led to more than $73 \%$ of surviving trees. Relevant incompatibility symptoms were not observed for 19 rootstocks that were selected due to overall good performance in 2009-2015, since all evaluated trees presented visual scores ranging from 1 to 2 (Table 3 ).

Table 3. Tolerance to drought (DT) (mean of 2010-2017, except 2015), tree survival rate until 2018 (SR), alternate bearing index (ABI, 2009-2015) and graft incompatibility (GI, 2017) of Valencia sweet orange [Citrus $\times$ sinensis (L.) Osbeck] grafted onto 46 rootstocks in rainfed cultivation under tropical savannah climate (Aw type) in Colombia, Northern Sao Paulo State, Brazil

\begin{tabular}{|c|c|c|c|c|c|c|c|c|}
\hline \multirow{2}{*}{ Rootstock } & \multirow{2}{*}{\multicolumn{2}{|c|}{$\mathrm{DT}^{1}$}} & \multirow{2}{*}{\multicolumn{2}{|c|}{ SR $(\%)$}} & \multirow{2}{*}{\multicolumn{2}{|c|}{$\mathrm{ABI}^{2}$}} & \multicolumn{2}{|c|}{$\mathrm{GI}^{3}(\%)$} \\
\hline & & & & & & & 1 & 2 \\
\hline Indio citrandarin & 1.91 & $\mathrm{~b}$ & 93.3 & $\mathrm{a}$ & 0.29 & $\mathrm{c}$ & 0 & 100 \\
\hline Riverside citrandarin & 1.96 & a & 86.7 & $\mathrm{a}$ & 0.38 & $\mathrm{~b}$ & 0 & 100 \\
\hline San Diego citrandarin & 1.99 & a & 100 & $\mathrm{a}$ & 0.31 & $\mathrm{c}$ & 0 & 100 \\
\hline CLEO $\times$ CTCZ-226 & 1.52 & $\mathrm{c}$ & 93.3 & a & 0.29 & $\mathrm{c}$ & - & - \\
\hline CLEO $\times$ TR Rubidoux-CO & 1.49 & $\mathrm{c}$ & 93.3 & $\mathrm{a}$ & 0.32 & $\mathrm{c}$ & - & - \\
\hline HTR-051 & 1.78 & $\mathrm{~b}$ & 100 & $\mathrm{a}$ & 0.33 & $\mathrm{c}$ & 0 & 100 \\
\hline HTR-053 & 2.05 & $\mathrm{a}$ & 100 & a & 0.36 & $\mathrm{c}$ & 0 & 100 \\
\hline HTR-069 & 1.85 & $\mathrm{~b}$ & 60.0 & $\mathrm{~b}$ & 0.39 & $\mathrm{~b}$ & - & - \\
\hline HTR-116 & 1.46 & $\mathrm{c}$ & 86.7 & $\mathrm{a}$ & 0.37 & $\mathrm{~b}$ & 33.3 & 66.7 \\
\hline $\mathrm{LCR} \times \mathrm{TR}-001$ & 2.23 & a & 86.7 & $\mathrm{a}$ & 0.38 & $\mathrm{~b}$ & - & - \\
\hline LCREEL $\times$ CTSW-001 & 1.36 & $\mathrm{c}$ & 86.7 & $\mathrm{a}$ & 0.32 & $\mathrm{c}$ & 0 & 100 \\
\hline CNPMF-003 Rangpur lime & 2.08 & a & 93.3 & a & 0.31 & $\mathrm{c}$ & 0 & 100 \\
\hline CNPMF-004 Rangpur lime & 2.15 & a & 93.3 & $\mathrm{a}$ & 0.33 & $\mathrm{c}$ & 16.7 & 83.3 \\
\hline Santa Cruz Rangpur lime & 2.03 & a & 46.7 & $\mathrm{~b}$ & 0.31 & $\mathrm{c}$ & 0 & 100 \\
\hline $\mathrm{LRF} \times(\mathrm{LCR} \times \mathrm{TR})-005$ & 1.87 & $\mathrm{~b}$ & 93.3 & a & 0.25 & $\mathrm{c}$ & 0 & 100 \\
\hline LVK $\times$ LCR-010 & 1.77 & $\mathrm{~b}$ & 73.3 & $\mathrm{a}$ & 0.34 & $\mathrm{c}$ & - & - \\
\hline LVK $\times$ LCR-038 & 2.03 & $\mathrm{a}$ & 93.3 & $\mathrm{a}$ & 0.30 & $\mathrm{c}$ & 83.3 & 16.7 \\
\hline LVK $\times$ LVA-009 & 1.77 & $\mathrm{~b}$ & 13.3 & $\mathrm{c}$ & 0.40 & $\mathrm{~b}$ & - & - \\
\hline TSK $\times$ Alemow-CO & 1.04 & d & 93.3 & $\mathrm{a}$ & 0.34 & $\mathrm{c}$ & - & - \\
\hline TSK × TR English-CO & 1.93 & $\mathrm{~b}$ & 100 & a & 0.34 & $\mathrm{c}$ & 0 & 100 \\
\hline Maravilha Sunki mandarin & 1.08 & $d$ & 53.3 & $\mathrm{~b}$ & 0.33 & $\mathrm{c}$ & - & - \\
\hline Tropical Sunki mandarin & 1.97 & $\mathrm{a}$ & 86.7 & $\mathrm{a}$ & 0.56 & $\mathrm{a}$ & 16.7 & 83.3 \\
\hline TSK $\times$ CTTR-002 & 2.16 & a & 60.0 & $\mathrm{~b}$ & 0.45 & $\mathrm{~b}$ & - & - \\
\hline TSK × TR Benecke-CO & 1.92 & $\mathrm{~b}$ & 93.3 & a & 0.33 & $\mathrm{c}$ & 0 & 100 \\
\hline $\mathrm{TSKC} \times(\mathrm{LCR} \times \mathrm{TR})-001$ & 1.51 & $\mathrm{c}$ & 100 & a & 0.34 & $\mathrm{c}$ & - & - \\
\hline $\mathrm{TSKC} \times(\mathrm{LCR} \times \mathrm{TR})-017$ & 1.96 & $\mathrm{a}$ & 100 & $\mathrm{a}$ & 0.40 & $\mathrm{~b}$ & 0 & 100 \\
\hline $\mathrm{TSKC} \times(\mathrm{LCR} \times \mathrm{TR})-018$ & 1.48 & $\mathrm{c}$ & 93.3 & a & 0.27 & $\mathrm{c}$ & - & - \\
\hline $\mathrm{TSKC} \times(\mathrm{LCR} \times \mathrm{TR})-059$ & 2.22 & a & 100 & a & 0.32 & $\mathrm{c}$ & 16.7 & 83.3 \\
\hline $\mathrm{TSKC} \times(\mathrm{LCR} \times \mathrm{TR})-073$ & 1.67 & $\mathrm{~b}$ & 66.7 & $\mathrm{~b}$ & 0.39 & $\mathrm{~b}$ & - & - \\
\hline TSKC $\times$ CTARG-001 & 1.23 & $d$ & 93.3 & $\mathrm{a}$ & 0.33 & $\mathrm{c}$ & - & - \\
\hline TSKC × CTARG-036 & 1.29 & d & 93.3 & a & 0.43 & $\mathrm{~b}$ & - & - \\
\hline TSKC $\times$ CTQT 1434-010 & 1.79 & $\mathrm{~b}$ & 86.7 & $\mathrm{a}$ & 0.33 & $\mathrm{c}$ & - & - \\
\hline TSKC $\times$ CTQT 1439-004 & 1.70 & $\mathrm{~b}$ & 86.7 & $\mathrm{a}$ & 0.38 & $\mathrm{~b}$ & - & - \\
\hline TSKC $\times$ CTQT 1439-026 & 1.19 & d & 86.7 & $\mathrm{a}$ & 0.32 & $\mathrm{c}$ & - & - \\
\hline TSKC $\times$ CTRK-001 & 1.07 & d & 40.0 & $\mathrm{~b}$ & 0.27 & $\mathrm{c}$ & - & - \\
\hline TSKC $\times$ CTSW-019 & 1.27 & d & 93.3 & $\mathrm{a}$ & 0.36 & $\mathrm{c}$ & - & - \\
\hline TSKC $\times$ CTSW -028 & 2.02 & $\mathrm{a}$ & 100 & $\mathrm{a}$ & 0.33 & $\mathrm{c}$ & 0 & 100 \\
\hline TSKC $\times$ CTSW-033 & 1.46 & $\mathrm{c}$ & 100 & a & 0.36 & $\mathrm{c}$ & - & - \\
\hline TSKC $\times$ CTSW -041 & 2.08 & $\mathrm{a}$ & 86.7 & $\mathrm{a}$ & 0.37 & $\mathrm{~b}$ & 0 & 100 \\
\hline TSKC $\times$ CTSW -064 & 1.35 & $\mathrm{c}$ & 80.0 & $\mathrm{a}$ & 0.35 & $\mathrm{c}$ & - & - \\
\hline TSKC $\times$ LHA-006 & 1.50 & $\mathrm{c}$ & 86.7 & $\mathrm{a}$ & 0.43 & $\mathrm{~b}$ & - & - \\
\hline TSKC $\times$ LHA-011 & 1.58 & $\mathrm{c}$ & 100 & $\mathrm{a}$ & 0.41 & $\mathrm{~b}$ & - & - \\
\hline
\end{tabular}




\begin{tabular}{llllll}
\hline TSKFL $\times$ CTC 25-010 & $1.46 \mathrm{c}$ & $53.3 \mathrm{~b}$ & $0.35 \mathrm{c}$ & - & - \\
$\mathrm{TSKFL} \times \mathrm{CTTR}-008$ & $1.22 \mathrm{~d}$ & $40.0 \mathrm{~b}$ & $0.40 \mathrm{~b}$ & - & - \\
$\mathrm{TSKFL} \times \mathrm{CTTR}-012$ & $1.65 \mathrm{c}$ & $93.3 \mathrm{a}$ & $0.30 \mathrm{c}$ & - & - \\
$\mathrm{TSKFL} \times \mathrm{CTTR}-022$ & $1.19 \mathrm{~d}$ & $100 \mathrm{a}$ & $0.29 \mathrm{c}$ & - & - \\
$\mathrm{P}$ & $* *$ & $* *$ & 20.71 & - & - \\
$\mathrm{CV}(\%)$ & 10.37 & 19.64 & 0.35 & - & - \\
Mean & 1.68 & 83.91 & 0.35 & - \\
\hline
\end{tabular}

Note. Means followed by the same letters in the column belong to the same group by the Scott-Knott test. (ns) not significant, $\left({ }^{*}\right)$ significant at $P<0.05,\left(^{* *}\right)$ significant at $P<0.01$ and $(* * *)$ significant at $P<0.15$. ${ }^{1}$ Tolerance to drought was assessed by visual scoring as adapted from Fadel et al. (2018): 1) low tolerance (leaf drop, shoot dieback and general leaf wilting in the canopy); 2) intermediate tolerance (partial leaf wilting in the canopy); and 3) high tolerance (no leaf wilting in the canopy). ${ }^{2}$ Estimated according to Pearce and Dobersek-Urbanc (1967). ${ }^{3}$ Percentage of trees classified by the visual scores adapted from Fadel et al. (2019): 1) no symptoms of graft incompatibility; 2) fine line separating the scion and the rootstock tissues; 3 ) marked line separating the scion and the rootstock tissues; and 4) sharp and deep line separating the scion and the rootstock tissues with rootstock phloem yellowing, necrosis and gum exudation in the graft union. Scores 3 and 4 were not observed. (-) not evaluated.

\subsection{Fruit Quality}

The evaluated rootstocks were grouped in five classes regarding the fruit weight induced to the Valencia sweet orange. In general, the lightest the fruit, the highest the soluble solids concentration in the juice. Nevertheless, four hybrids resulted in the largest fruits that are valued by the fresh market. Rootstocks that induced the highest juice content included all selections of Rangpur lime, Tropical Sunki mandarin, most citrandarins, and CTQT hybrids among others. Citrandarins introduced from Colombia (CLEO $\times$ TR Rubidoux, TSK $\times$ TR English and TSK $\times$ TR Benecke) stood out for the highest concentration of soluble solids (SS) induced to the Valencia fruits, increasing in average $2.0^{\circ} \mathrm{Brix}$ in relation to all selections of Rangpur lime. Other rootstocks that were the most productive induced intermediate SS. Rangpur lime selections were also in the group inducing the lowest titratable acidity (TA), whereas TSK $\times$ TR Benecke-CO, TSK $\times$ TR English-CO, San Diego and Riverside citrandarins, and TSKC $\times$ CTSW-028 are highlighted within the rootstocks inducing higher TA. About half of the evaluated rootstocks led to higher maturity index than the standard Santa Cruz Rangpur lime, indicating an earlier fruit maturation, which was notable for some productive semi-dwarfing rootstocks like HTR-051 and-053, $\mathrm{LCR} \times \mathrm{TR}-001, \mathrm{LRF} \times(\mathrm{LCR} \times \mathrm{TR})-005$, and TSKC $\times(\mathrm{LCR} \times \mathrm{TR})-017$ and-059. Rangpur lime selections were grouped within the lowest mean technological index, which was $16.7 \%$ lower in relation to that induced by the citrandarins introduced from Colombia (Table 4).

Table 4. Mean fruit weight (FW), juice content (JC), soluble solids concentration (SS), titratable acidity (TA), maturity index (MI, SS/TA) and technological index (TI) of fruits of Valencia sweet orange [Citrus $\times$ sinensis (L.) Osbeck] grafted onto 46 rootstocks in 2009-2015 in rainfed cultivation under tropical savannah climate (Aw type) in Colombia, Northern Sao Paulo State, Brazil

\begin{tabular}{|c|c|c|c|c|c|c|c|c|c|c|c|c|}
\hline Rootstock & $\mathrm{FW}(\mathrm{g})$ & & $\mathrm{JC}(\%)$ & & $\mathrm{SS}\left({ }^{\circ} \mathrm{B}\right.$ & (rix) & TA $(\%$ & & $\mathrm{MI}(\mathrm{S}$ & /TA) & $\mathrm{TI}^{1}$ & \\
\hline Indio citrandarin & 193.77 & $\mathrm{c}$ & 48.01 & $\mathrm{a}$ & 11.58 & $\mathrm{c}$ & 0.71 & $\mathrm{~b}$ & 16.44 & $\mathrm{~b}$ & 2.24 & $\mathrm{~b}$ \\
\hline Riverside citrandarin & 198.97 & $\mathrm{c}$ & 46.50 & $\mathrm{~b}$ & 11.65 & $\mathrm{c}$ & 0.74 & a & 15.80 & $\mathrm{~b}$ & 2.18 & $\mathrm{c}$ \\
\hline San Diego citrandarin & 200.41 & $\mathrm{c}$ & 49.23 & $\mathrm{a}$ & 11.38 & $\mathrm{c}$ & 0.76 & $\mathrm{a}$ & 15.56 & $\mathrm{~b}$ & 2.32 & $\mathrm{~b}$ \\
\hline CLEO × CTCZ-226 & 194.94 & $\mathrm{c}$ & 45.40 & $\mathrm{~b}$ & 11.77 & $\mathrm{c}$ & 0.64 & $\mathrm{c}$ & 18.53 & a & 2.15 & $\mathrm{c}$ \\
\hline CLEO $\times$ TR Rubidoux-CO & 152.93 & f & 47.93 & $\mathrm{a}$ & 12.99 & $\mathrm{a}$ & 0.70 & $\mathrm{~b}$ & 19.09 & a & 2.55 & $\mathrm{a}$ \\
\hline HTR-051 & 205.06 & $\mathrm{c}$ & 45.21 & $\mathrm{~b}$ & 11.58 & $\mathrm{c}$ & 0.64 & $\mathrm{c}$ & 18.38 & a & 2.14 & $\mathrm{c}$ \\
\hline HTR-053 & 224.72 & $\mathrm{a}$ & 45.94 & $\mathrm{~b}$ & 11.27 & $\mathrm{c}$ & 0.64 & c & 17.78 & $\mathrm{a}$ & 2.08 & $\mathrm{c}$ \\
\hline HTR-069 & 219.38 & a & 46.05 & $\mathrm{~b}$ & 10.58 & $\mathrm{~d}$ & 0.56 & $\mathrm{c}$ & 18.99 & $\mathrm{a}$ & 2.01 & $\mathrm{c}$ \\
\hline HTR-116 & 227.05 & a & 43.80 & $\mathrm{~b}$ & 11.32 & $\mathrm{c}$ & 0.61 & $\mathrm{c}$ & 18.29 & a & 2.00 & $\mathrm{c}$ \\
\hline $\mathrm{LCR} \times \mathrm{TR}-001$ & 220.27 & $\mathrm{a}$ & 47.15 & $\mathrm{a}$ & 10.94 & $\mathrm{~d}$ & 0.60 & $\mathrm{c}$ & 18.49 & $\mathrm{a}$ & 2.13 & $\mathrm{c}$ \\
\hline LCREEL × CTSW-001 & 207.33 & $\mathrm{~b}$ & 44.82 & $\mathrm{~b}$ & 12.07 & $\mathrm{~b}$ & 0.67 & $\mathrm{c}$ & 18.00 & a & 2.17 & $\mathrm{c}$ \\
\hline CNPMF-003 Rangpur lime & 198.15 & $\mathrm{c}$ & 47.10 & $\mathrm{a}$ & 10.81 & $\mathrm{~d}$ & 0.62 & $\mathrm{c}$ & 17.71 & a & 2.11 & $\mathrm{c}$ \\
\hline CNPMF-004 Rangpur lime & 201.54 & $\mathrm{c}$ & 47.36 & $\mathrm{a}$ & 11.23 & $\mathrm{~d}$ & 0.63 & $\mathrm{c}$ & 17.43 & $\mathrm{~b}$ & 2.10 & $\mathrm{c}$ \\
\hline Santa Cruz Rangpur lime & 202.85 & $\mathrm{c}$ & 49.07 & $\mathrm{a}$ & 10.85 & $\mathrm{~d}$ & 0.63 & $\mathrm{c}$ & 16.70 & $\mathrm{~b}$ & 2.09 & $\mathrm{c}$ \\
\hline
\end{tabular}




\begin{tabular}{|c|c|c|c|c|c|c|c|c|c|c|c|c|}
\hline $\mathrm{LRF} \times(\mathrm{LCR} \times \mathrm{TR})-005$ & 170.23 & $\mathrm{e}$ & 47.22 & $\mathrm{a}$ & 11.94 & $\mathrm{~b}$ & 0.65 & $\mathrm{c}$ & 18.47 & $\mathrm{a}$ & 2.28 & $\mathrm{~b}$ \\
\hline LVK $\times$ LCR -010 & 193.97 & $\mathrm{c}$ & 44.98 & $\mathrm{~b}$ & 10.99 & $\mathrm{~d}$ & 0.66 & $\mathrm{c}$ & 16.66 & $\mathrm{~b}$ & 2.00 & $\mathrm{c}$ \\
\hline LVK $\times$ LCR-038 & 206.62 & $\mathrm{~b}$ & 44.12 & $\mathrm{~b}$ & 11.37 & $\mathrm{c}$ & 0.65 & $\mathrm{c}$ & 17.40 & $\mathrm{~b}$ & 2.00 & $\mathrm{c}$ \\
\hline LVK $\times$ LVA-009 & 182.06 & d & 44.21 & $\mathrm{~b}$ & 12.39 & $\mathrm{~b}$ & 0.70 & b & 16.72 & $\mathrm{~b}$ & 2.09 & $\mathrm{c}$ \\
\hline TSK $\times$ Alemow-CO & 177.37 & $\mathrm{~d}$ & 45.65 & $\mathrm{~b}$ & 11.34 & $\mathrm{c}$ & 0.64 & $\mathrm{c}$ & 19.19 & $\mathrm{a}$ & 2.23 & b \\
\hline TSK $\times$ TR English-CO & 164.90 & $\mathrm{e}$ & 47.85 & $\mathrm{a}$ & 12.88 & a & 0.75 & $\mathrm{a}$ & 17.31 & $\mathrm{~b}$ & 2.34 & b \\
\hline Maravilha Sunki mandarin & 183.55 & $\mathrm{~d}$ & 45.80 & $\mathrm{~b}$ & 11.79 & $\mathrm{c}$ & 0.71 & b & 16.73 & $\mathrm{~b}$ & 2.16 & $\mathrm{c}$ \\
\hline Tropical Sunki mandarin & 211.47 & $\mathrm{~b}$ & 48.09 & $\mathrm{a}$ & 11.52 & $\mathrm{c}$ & 0.69 & $\mathrm{~b}$ & 16.44 & $\mathrm{~b}$ & 2.18 & $\mathrm{c}$ \\
\hline TSK $\times$ CTTR-002 & 204.56 & c & 44.99 & $\mathrm{~b}$ & 11.15 & d & 0.61 & $\mathrm{c}$ & 18.59 & a & 2.05 & $\mathrm{c}$ \\
\hline TSK $\times$ TR Benecke-CO & 155.65 & $f$ & 48.10 & $\mathrm{a}$ & 12.50 & a & 0.80 & $\mathrm{a}$ & 16.08 & $\mathrm{~b}$ & 2.49 & $\mathrm{a}$ \\
\hline $\mathrm{TSKC} \times(\mathrm{LCR} \times \mathrm{TR})-001$ & 206.96 & $\mathrm{~b}$ & 46.48 & $\mathrm{~b}$ & 11.75 & $\mathrm{c}$ & 0.65 & $\mathrm{c}$ & 18.05 & a & 2.19 & $\mathrm{c}$ \\
\hline $\mathrm{TSKC} \times(\mathrm{LCR} \times \mathrm{TR})-017$ & 212.51 & $\mathrm{~b}$ & 47.43 & $\mathrm{a}$ & 11.58 & $\mathrm{c}$ & 0.61 & $\mathrm{c}$ & 18.10 & $\mathrm{a}$ & 2.15 & $\mathrm{c}$ \\
\hline $\mathrm{TSKC} \times(\mathrm{LCR} \times \mathrm{TR})-018$ & 198.93 & $\mathrm{c}$ & 45.25 & $\mathrm{~b}$ & 11.04 & $\mathrm{~d}$ & 0.66 & $\mathrm{c}$ & 16.67 & $\mathrm{~b}$ & 2.03 & $\mathrm{c}$ \\
\hline $\mathrm{TSKC} \times(\mathrm{LCR} \times \mathrm{TR})-059$ & 210.00 & $\mathrm{~b}$ & 47.68 & $\mathrm{a}$ & 12.02 & $\mathrm{~b}$ & 0.69 & $\mathrm{~b}$ & 17.67 & a & 2.31 & $\mathrm{~b}$ \\
\hline $\mathrm{TSKC} \times(\mathrm{LCR} \times \mathrm{TR})-073$ & 196.75 & $\mathrm{c}$ & 45.45 & $\mathrm{~b}$ & 11.67 & $\mathrm{c}$ & 0.66 & $\mathrm{c}$ & 17.83 & $\mathrm{a}$ & 2.15 & $\mathrm{c}$ \\
\hline TSKC $\times$ CTARG-001 & 200.63 & $\mathrm{c}$ & 44.42 & $\mathrm{~b}$ & 11.67 & $\mathrm{c}$ & 0.75 & $\mathrm{a}$ & 15.57 & $\mathrm{~b}$ & 2.06 & $\mathrm{c}$ \\
\hline TSKC $\times$ CTARG-036 & 200.83 & $\mathrm{c}$ & 45.58 & $\mathrm{~b}$ & 11.47 & $\mathrm{c}$ & 0.64 & $\mathrm{c}$ & 18.51 & a & 2.15 & $\mathrm{c}$ \\
\hline TSKC $\times$ CTQT 1434-010 & 201.81 & $\mathrm{c}$ & 48.72 & $\mathrm{a}$ & 11.94 & $\mathrm{~b}$ & 0.65 & $\mathrm{c}$ & 18.26 & $\mathrm{a}$ & 2.34 & b \\
\hline TSKC $\times$ CTQT 1439-004 & 180.56 & d & 48.51 & $\mathrm{a}$ & 11.55 & $\mathrm{c}$ & 0.69 & b & 17.14 & $\mathrm{~b}$ & 2.31 & $\mathrm{~b}$ \\
\hline TSKC $\times$ CTQT 1439-026 & 181.97 & $\mathrm{~d}$ & 48.14 & $\mathrm{a}$ & 12.23 & $\mathrm{~b}$ & 0.64 & $\mathrm{c}$ & 18.76 & $\mathrm{a}$ & 2.32 & $\mathrm{~b}$ \\
\hline TSKC $\times$ CTRK-001 & 189.32 & $\mathrm{c}$ & 47.03 & $\mathrm{a}$ & 12.06 & $\mathrm{~b}$ & 0.69 & b & 17.93 & $\mathrm{a}$ & 2.30 & b \\
\hline TSKC $\times$ CTSW-019 & 186.83 & d & 43.82 & $\mathrm{~b}$ & 11.51 & $\mathrm{c}$ & 0.73 & $\mathrm{a}$ & 16.25 & $\mathrm{~b}$ & 2.09 & $\mathrm{c}$ \\
\hline TSKC $\times$ CTSW -028 & 197.94 & $\mathrm{c}$ & 47.30 & $\mathrm{a}$ & 11.68 & $\mathrm{c}$ & 0.73 & $\mathrm{a}$ & 16.12 & $\mathrm{~b}$ & 2.24 & $\mathrm{~b}$ \\
\hline TSKC $\times$ CTSW -033 & 191.67 & $\mathrm{c}$ & 48.27 & $\mathrm{a}$ & 11.59 & $\mathrm{c}$ & 0.68 & $\mathrm{~b}$ & 17.32 & $\mathrm{~b}$ & 2.30 & $\mathrm{~b}$ \\
\hline TSKC $\times$ CTSW -041 & 212.51 & $\mathrm{~b}$ & 47.96 & $\mathrm{a}$ & 11.29 & $\mathrm{c}$ & 0.66 & $\mathrm{c}$ & 17.39 & $\mathrm{~b}$ & 2.23 & $\mathrm{~b}$ \\
\hline TSKC $\times$ CTSW-064 & 191.91 & $\mathrm{c}$ & 45.92 & $\mathrm{~b}$ & 11.45 & $\mathrm{c}$ & 0.69 & b & 16.93 & $\mathrm{~b}$ & 2.16 & $\mathrm{c}$ \\
\hline TSKC $\times$ LHA-006 & 194.58 & $\mathrm{c}$ & 48.15 & $\mathrm{a}$ & 11.69 & $\mathrm{c}$ & 0.73 & a & 16.28 & $\mathrm{~b}$ & 2.27 & $\mathrm{~b}$ \\
\hline TSKC $\times$ LHA- 011 & 191.84 & $\mathrm{c}$ & 45.00 & $\mathrm{~b}$ & 11.98 & $\mathrm{~b}$ & 0.72 & $\mathrm{a}$ & 16.95 & $\mathrm{~b}$ & 2.20 & $\mathrm{c}$ \\
\hline TSKFL $\times$ CTC $25-010$ & 201.45 & $\mathrm{c}$ & 45.11 & $\mathrm{~b}$ & 11.42 & $\mathrm{c}$ & 0.58 & $\mathrm{c}$ & 19.76 & $\mathrm{a}$ & 2.07 & $\mathrm{c}$ \\
\hline TSKFL $\times$ CTTR -008 & 184.13 & $\mathrm{~d}$ & 43.80 & $\mathrm{~b}$ & 12.09 & $\mathrm{~b}$ & 0.75 & $\mathrm{a}$ & 16.83 & $\mathrm{~b}$ & 2.22 & $\mathrm{~b}$ \\
\hline TSKFL $\times$ CTTR -012 & 210.88 & $\mathrm{~b}$ & 46.40 & $\mathrm{~b}$ & 10.98 & d & 0.64 & $\mathrm{c}$ & 17.17 & $\mathrm{~b}$ & 2.05 & $\mathrm{c}$ \\
\hline TSKFL $\times$ CTTR -022 & 191.59 & $\mathrm{c}$ & 47.55 & $\mathrm{a}$ & 11.56 & $\mathrm{c}$ & 0.59 & $\mathrm{c}$ & 20.17 & a & 2.26 & $\mathrm{~b}$ \\
\hline $\mathrm{P}$ & $* *$ & & $* *$ & & $* *$ & & $* *$ & & $* *$ & & $* *$ & \\
\hline $\mathrm{CV}(\%)$ & 4.14 & & 4.13 & & 3.37 & & 6.42 & & 5.71 & & 5.50 & \\
\hline Mean & 196.38 & & 46.5 & & 11.61 & & 0.67 & & 17.53 & & 2.18 & \\
\hline
\end{tabular}

Note. Means followed by the same letters in the column belong to the same group by the Scott-Knott test. (ns) not significant, $\left({ }^{*}\right)$ significant at $P<0.05$ and (**) significant at $P<0.01 .{ }^{1}$ Expressed as $\mathrm{kg}$ of SS per $40.8 \mathrm{~kg}$ of fruit (standard orange box).

\subsection{Multivariate Analyses}

The principal component analysis (PCA) indicated the presence of genetic variation among the evaluated rootstocks (Figure 3). Two principal components represented $68.07 \%$ of total variation, with PC1 responsible for $43.30 \%$ and PC2 for $24.77 \%$ of data variation. The fruit yield $(31.59 \%)$ and tolerance to drought $(31.39 \%)$ contributed the most for PC1, while PC2 was explained mainly by the production efficiency $(36.25 \%)$ and plant height (51.97\%) (Figure 3A). There was a positive relation between the fruit yield, tolerance to drought and juice content, which in turn were inversely related to the soluble solids concentration, whereas plant height was inversely related to the production efficiency (Figure 3B). 
(A)

\begin{tabular}{|c|c|c|c|}
\hline & & \multicolumn{2}{|c|}{ Principal component } \\
\hline & & CP1 & $\mathrm{CP} 2$ \\
\hline \multirow{6}{*}{$\begin{array}{l}\overline{\frac{0}{0}} \\
\frac{0}{3} \\
3\end{array}$} & Yield & 31.59 & 4.69 \\
\hline & EfP & 14.39 & 36.25 \\
\hline & SS & 11.85 & 5.58 \\
\hline & $\mathrm{JC}$ & 6.83 & 1.44 \\
\hline & DT & 31.39 & 0.06 \\
\hline & Height & 3.94 & 51.97 \\
\hline
\end{tabular}

(B)

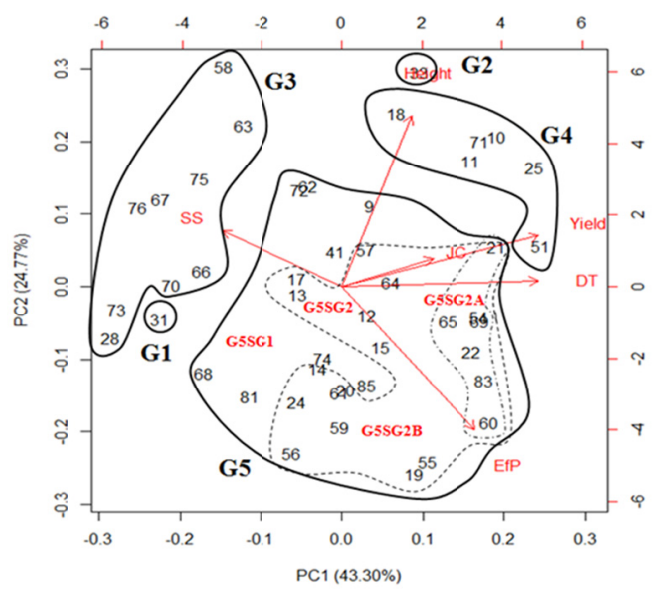

Groups
_
_ . - . - Subgroups
(C) Link distance

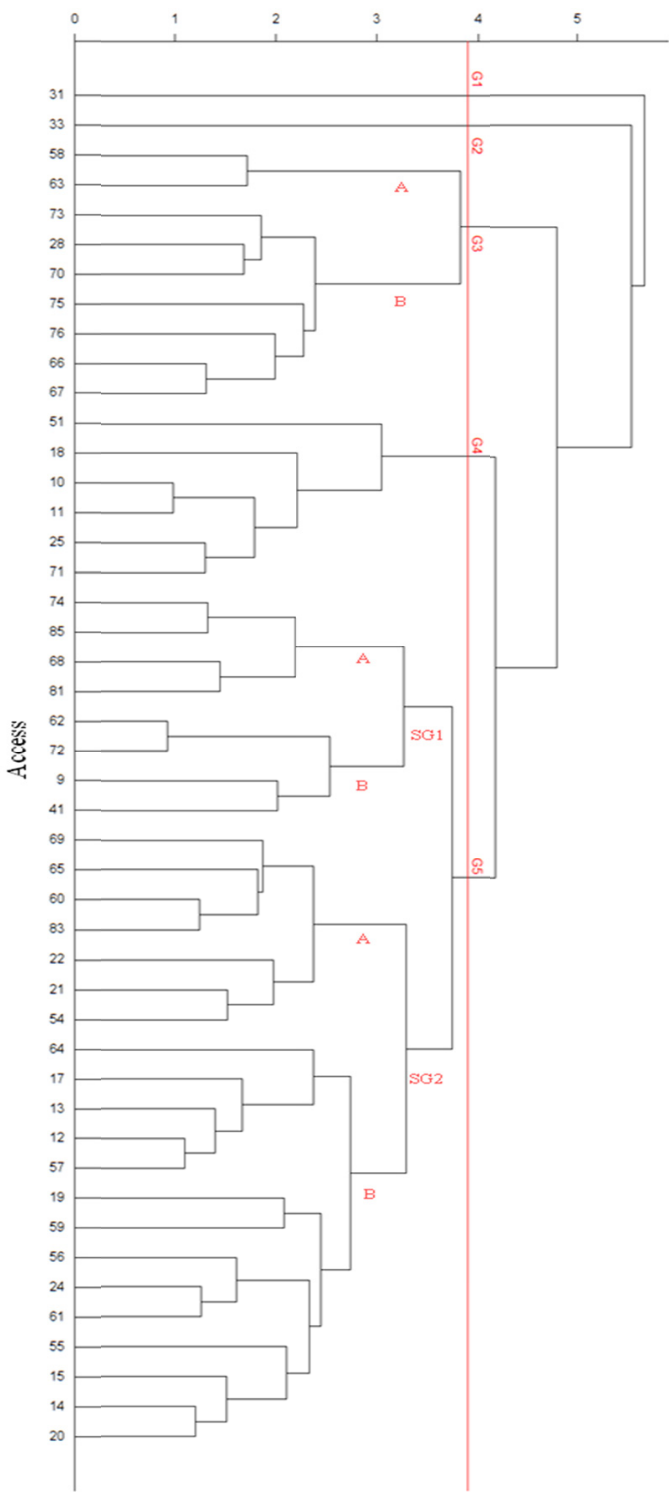

Figure 3. Weighting coefficients of variables obtained by the UPGMA method (Unweighted Pair Group Method using Arithmetic averages) (A), score graphs of the principal components analysis (B) and dendogram (C) using the dissimilarity measurements of the variables: mean fruit yield (FY), mean production efficiency (Efp), juice content (JC), tolerance to drought based on visual symptoms scoring (DT), plant height (H) and soluble solids concentration in the juice (SS) of Valencia sweet orange grafted onto 46 rootstocks in 2009-2015 in rainfed cultivation under tropical savannah climate (Aw type) in Colombia, Northern Sao Paulo State, Brazil. CCC = 0.71**. G1: 31 (CLEO $\times$ TR Rubidoux-CO). G2: 33 (TSK $\times$ TR English Palmira-CO). G3A: 58 (TSKC $\times$ CTARG-001), $63($ TSKC $\times$ CTSW-019). G3B: $73($ TSKFL $\times$ CTTR-008), $28($ TSK $\times$ Alemow-CO), 70 $($ LCREEL $\times$ CTSW-001), $75($ TSKC $\times$ LHA-011), $76($ TSKC $\times$ CTRK-001), $66($ TSKC $\times$ CTARG-036), 67

(Maravilha Sunki mandarin). G4: 51 (Santa Cruz Rangpur lime), 18 (Riverside citrandarin), 10 (Indio citrandarin), 11 (TSKC $\times$ CTSW-028), 25 (CNPMF-004 Rangpur lime), 71 (Tropical Sunki mandarin). G5SG1:

$74($ TSKC $\times$ CTSW-033), $85($ TSKC $\times$ CTQT 1434-010), $68($ TSKFL $\times$ CTTR-022), $81($ TSKC $\times$ CTQT 1439-026), $62($ TSKC $\times$ CTQT 1439-004), $72($ TSKC $\times$ LHA-006), $9($ LRF $\times($ LCR $\times$ TR $)-005), 41($ TSK $\times$ TR Benecke-CO). G5SG2A: 69 (HTR-053), 65 (CNPMF-003 Rangpur lime), 60 (LCR $\times$ TR-001), 83 (TSKC $\times$

$(\mathrm{LCR} \times \mathrm{TR})-017), 22($ TSKC $\times(\mathrm{LCR} \times \mathrm{TR})-059), 21$ (San Diego citrandarin), $54($ TSKC $\times$ CTSW-041). G5SG2B: 64 (TSK $\times$ CTTR-002), 17 (LVK $\times$ LVA-009), $13($ CLEO $\times$ CTCZ-226), 12 (HTR-051), 57 (TSKC $\times$ $(\mathrm{LCR} \times \mathrm{TR})-073), 19(\mathrm{LVK} \times \mathrm{LCR}-038), 59(\mathrm{HTR}-116), 56(\mathrm{TSKFL} \times \mathrm{CTC} 25-010), 24(\mathrm{TSKC} \times \mathrm{CTSW}-064)$, $61(\mathrm{TSKC} \times(\mathrm{LCR} \times \mathrm{TR})-001), 55(\mathrm{HTR}-069), 15(\mathrm{LVK} \times \mathrm{LCR}-010), 14(\mathrm{TSKC} \times(\mathrm{LCR} \times \mathrm{TR})-018)$, $20($ TSKFL $\times$ CTTR-012) 
The cofenetic correlation was significant $\left(\mathrm{r}=0.71^{* *}\right)$, and the rootstocks were sorted in five groups $(\mathrm{G})$ according to the similarity patterns (Figure 3C). The citrandarin CLEO $\times$ TR Rubidoux-CO is the unique rootstock in $\mathrm{G} 1$, and it is characterized by the highest SS, but poor performance related to the other variables. The citrandarin TSK $\times$ TR English-CO represents G2, presenting high quality of juice, high fruit yield, large trees and intermediate tolerance to drought. G3 can be divided in G3A, comprising very vigorous rootstocks with poor performance overall, and G3B with the same characteristics except by the lower tree size, such as Maravilha Sunki mandarin. Drought-tolerant rootstocks that induce high fruit yield with high juice content and varying SS, large tree size and intermediate production efficiency are grouped within G4: Santa Cruz and CNPMF-004 Rangpur limes, Riverside and Indio citrandarins, TSKC $\times$ CTSW-028 and Tropical Sunki mandarin G5 was subdivided into G5SG1 and G5SG2, which in turn were also subdivided to facilitate understanding. G5SG1A includes four hybrids that induce low yield, low tolerance to drought and small trees, yet high juice content. G5SG1B is similar except by the higher tree size, varying fruit yield and markedly higher SS. G5SG2 comprises the least vigorous rootstocks, with G5SG2A grouping those with the best performance, that is, high fruit yield, high tolerance to drought, good fruit quality, high production efficiency and smaller tree size: HTR-053, CNPMF-003 Rangpur lime, LCR $\times$ TR-001, TSKC $\times($ LCR $\times$ TR $)-017$ and-059, San Diego citrandarin and TSKC $\times$ CTSW-041. Finally, G5SG2B grouped the remaining rootstocks with intermediate to low means overall.

\section{Discussion}

Citrandarins presented outstanding performance in several trials in very different environments and constitute one of the most promising new hybrid rootstocks (Bowman, Faulkner, \& Kesinger, 2016; Schinor et al., 2013; Legua, Bellver, Forner, \& Forner-Giner et al., 2011). Some citrandarins introduced in Sao Paulo, Brazil, from the USA induced high fruit production to Valencia sweet orange (Costa et al., 2016; Pompeu Junior \& Blumer, 2011; Pompeu Junior et al., 2002). In this work, we evaluated 41 hybrid rootstocks, comprising citrandarins and other trifoliate hybrids that presented a competitive performance in relation to selections of Rangpur lime and Sunki mandarin in rainfed cultivation under tropical savannah climate (Aw type) conditions.

Indio, San Diego, Riverside and TSK $\times$ TR English-CO citrandarins induced large tree size and high production of good quality fruits to the Valencia sweet orange. They resulted from the Sunki mandarin $\times$ English trifoliate orange cross, excepted by San Diego, which is a hybrid of Sunki mandarin $\times$ Swingle trifoliate orange in a similar way to Bitters, Furr and Carpenter citrandarins (Siebert, Krueger, Kahn, Bash, \& Vidalakis, 2010). Hybrids of Sunki $\times$ English also stood out when grafted with Valencia sweet orange in the tropical lowlands of Colombia (Chaparro-Zambrano, Velázquez, \& Orduz-Rodríguez, 2015) and in different regions of Sao Paulo ranging from Cfa to Cwa climate types (Pompeu Junior \& Blumer, 2009, 2011; Blumer \& Pompeu Junior, 2005). Although TSK $\times$ TR Benecke and CLEO $\times$ TR Rubidoux citrandarins were previously indicated as highly productive in combination with Valencia in the south of Sao Paulo (Blumer \& Pompeu Junior, 2005; Pompeu Junior et al., 2002), in the warmer and drier north region their production and plant growth were poor, yet fruit quality was very high, suggesting a higher intolerance to water deficit. On the other hand, under tropical sub-humid climate with dry summer (As type) in Northeastern Brazil, Tuxpan Valencia sweet orange grafted onto Indio and Riverside citrandarins had similar fruit yield than on the Rangpur lime without irrigation, therefore, indicating their adaptability even under severe drought conditions (França, Amorim, Girardi, Passos, \& Soares Filho, 2016).

Other hybrids were also selected due to an additional high production efficiency, including one citromonia (LCR $\times$ TR-001), two citromoniandarins [TSKC $\times($ LCR $\times$ TR)-017 and-059], one trifoliate hybrid (HTR-053) and two citrumelandarins (TSKC $\times$ CTSW-028 and-041). The late four hybrids were registered in the National Register of Cultivars of the Ministry of Agriculture, Livestock and Supply of Brazil as BRS Bravo, BRS Passos, BRS Pompeu and BRS Cunha Sobrinho, respectively, aiming at future commercial release. The higher production efficiency was a result of the high fruit yield and a mean $30 \%$ decrease in tree size in relation to the most vigorous rootstocks, with the exception of TSKC $\times \mathrm{CTSW}-028$, but none rootstock could be classified as true dwarfing variety. Furthermore, two selections of Sunki mandarin were studied because this rootstock induces high yield, tree size and fruit quality to late season sweet oranges in rainfed cultivation (Girardi, Cerqueira, Cantuarias-Avilés, Silva, \& Stuchi, 2017). Tropical Sunki corroborated its superior performance and good tolerance to drought in field conditions compared to the Maravilha selection (Carvalho et al., 2019; França et al., 2016).

The fruit quality of sweet orange grafted onto trifoliate hybrids is usually higher in relation to trees grafted onto the Rangpur lime (Bowman \& Joubert, 2020), which was confirmed in this work. Moreover, the selected rootstocks induced mean fruit quality throughout the initial seven crops within the average range or slightly 
above according to historical data on Valencia sweet orange processing in the State of Sao Paulo (Nonino, 1995). Particularly, soluble solids concentration and ratio were high, which are important parameters for NFC juice. In addition, even though the cause of tree mortality was not confirmed in this study, visual symptoms of CSD were observed only for the Rangpur lime selections at eleven years after planting. LVK $\times$ LVA-009 seedlings were previously demonstrated to be highly intolerant to CTV (Rodrigues et al., 2014), which may be related to the highest rate of tree loss. None of the selected rootstocks presented graft incompatibility with Valencia sweet orange. The experimental area will be further investigated for the long-term responses of the selected rootstocks to citrus diseases.

Rangpur lime induced high production, low alternate bearing and moderate leaf wilting, which are all indicative of high tolerance to drought (Schinor et al., 2013; Fadel et al., 2018). A similar behavior was observed in most selected rootstocks, which was interestingly not related to the tree size class. The rootstock influences on the alternate bearing of the scion variety under water deficit conditions, with variations higher than $50 \%$ in some late sweet orange varieties (Yildiz, Demirkeser, \& Kaplankiran, 2013; Cantuarias-Avilés, Mourão Filho, Stuchi, Silva, \& Espinoza-Núñez, 2011). Considering the scenario of constraining climate change and limited water supply for citrus irrigation in the major producing regions (Fares, Bayabil, Zekri, Mattos Junior, \& Awal, 2017; Carr, 2012), the breeding of rootstocks that use water more efficiently or tolerate seasonal drought will become determinant for the sustainability of the citriculture on condition that high yield of high quality fruits will remain as main selection criteria.

\section{Conclusion}

Indio, Riverside, San Diego and TSK $\times$ TR English-CO citrandarins, Tropical Sunki mandarin and the hybrids TSKC $\times$ CTSW-028 and-041, HTR-053, LCR $\times$ TR-001 and TSKC $\times($ LCR $\times$ TR)-017 and-059 have potential as alternative rootstocks of Valencia sweet orange in rainfed cultivation under tropical savannah Aw type climate. These rootstocks induce higher fruit quality and tree survival rate, and similar fruit yield and tolerance to drought in relation to the standard Rangpur lime. The later five hybrids also lead to higher fruit production efficiency due to a $30 \%$ reduction in the canopy volume.

\section{Acknowledgements}

To the Coordenação de Aperfeiçoamento de Pessoal de Nível Superior (CAPES)-Financial Code 001, for the scholarship for the first author; to the Conselho Nacional de Desenvolvimento Científico e Tecnológico (CNPq) for the scholarship to ESS, ASG, WSSF, CASL and MACF; to the Citrosuco S/A Agroindústria and Muriti farm, for the experimental area and technical support; to the Fundação Coopercitrus Credicitrus (FCC), for technical support; to Embrapa Mandioca e Fruticultura (CNPMF) for plant material and financial aid (02.13.03.005.00.00 and 20.18.01.007.00.00); to Antônio Santana da Silva, Getúlio de Souza Vieira and Magno Guimarães Santos for their sound technical support; to Eng. Otávio Ricardo Sempionato, for suggestions relevant to the work.

\section{References}

Bassanezi, R. B., Bergamin Filho, A., Amorim, L., Gimenes-Fernandes, N., Gottwald, T. R., \& Bové, J. M. (2003). Spatial and temporal analyses of citrus sudden death, as a tool to generate hypotheses concerning its ethiology. Phytopathology, 93, 502-512. http://doi.org/10.1094/PHYTO.2003.93.4.502

Blumer, S., \& Pompeu Junior, J. (2005). Avaliação de citrandarins e outros híbridos de trifoliata como porta-enxertos para citros em São Paulo. Revista Brasileira de Fruticultura, 27, 264-267. http://doi.org/ $10.1590 / \mathrm{S} 0100-29452005000200019$

Bowman, K. D., \& Joubert, J. (2020). Citrus rootstocks. In M. Talon, M. Caruso, \& F. G. Gmitter Jr (Eds.), The Genus Citrus (pp. 105-128). Duxford: Woodhead Publishing. https://doi.org/10.1016/B978-0-12-812163-4. 00006-1

Bowman, K. D., Faulkner, L., \& Kesinger, M. (2016). New Citrus Rootstocks Released by USDA 2001-2010: Field Performance and Nursery Characteristics. HortScience, 51, 1208-1214. https://doi.org/10.21273/ HORTSCI10970-16

Cantuarias-Avilés, T., Mourão Filho, F. A. A., Stuchi, E. S., Silva, S. R., \& Espinoza-Núñez, E. (2011). Horticultural performance of Folha Murcha sweet orange onto twelve rootstocks. Scientia Horticulture, 29(2), 259-265. https://doi.org/10.1016/j.scienta.2011.03.039

Carr, M. K. V. (2012). The water relations and irrigation requirements of citrus (Citrus spp.): A review. Experimental Agriculture, 48, 347-377. https://doi.org/10.1017/S0014479712000038 
Carvalho, L. M., Carvalho, H. W. L., Barros, I., Martins, C. R., Soares Filho, W. S., Girardi, E. A., \& Passos, O. S. (2019). New scion-rootstock combinations for diversification of sweet orange orchards in tropical hardsetting soils. Scientia Horticulturae, 243, 169-176. https://doi.org/10.1016/j.scienta.2018.07.032

Carvalho, S. A., Girardi, E. A., Mourão Filho, F. A. A., Ferrarezi, R. S., \& Coletta Filho, H. D. (2019). Advances in citrus propagation in Brazil. Revista Brasileira de Fruticultura, 41(6), e-422. https://doi.org/10.1590/ 0100-29452019422

Castle, W. S. (2010). A career perspective on Citrus rootstocks, their development, and commercialization. HortScience, 45, 11-15. http://https://doi.org/10.21273/HORTSCI.45.1.11

Castle, W. S., \& Phillips, R. L. (1977). Potentially dwarfing rootstocks for Florida citrus. Proceeding International Society Citriculture, 2, 558-561. Retrieved May 15, 2020, from https://www.researchgate.net/ publication/272349840_Potentially_Dwarfing_Rootstocks_for_Florida_Citrus

Chaparro-Zambrano, H. N, Velázquez, H. A., \& Orduz-Rodríguez, J. O. (2015). Performance of Valencia sweet orange grafted in different rootstocks, Colombia Tropical Lowland, 2001-2013. Agronomia Colombiana, 33, 43-48. http://doi.org/10.15446/agron.colomb.v33n1.49497

Charrad, M., Ghazzali, N., Boiteau, V., \& Niknafs, A. (2013). NbClust: An examination of indices for determining the number of clusters. NbClust Package. Retrieved November 12, 2019, from https://cran.rproject.org/web/packages/NbClust/NbClust.pdf

Cimen, B., \& Yesiloglu, T. (2016) Rootstock Breeding for Abiotic Stress Tolerance in Citrus. In: A. Shanker, Abiotic and Biotic Stress in Plants-Recent Advances and Future Perspectives (pp. 527-563). London: Intech Open. http://doi.org/10.5772/62047

Costa, D. P., Stuchi, E. S., Girardi, E. A., Ramos, Y. C., Fadel, A. L., Maldonado Junior, W. M., ... Soares Filho, W. S. (2016). Potential rootstocks for Valencia sweet orange in rain-fed cultivation in the north of São Paulo, Brazil. Citrus Research \& Technology, 37, 26-36. http://doi.org/10.4322/crt.ICC038

Cruz, C. D., Regazzi, A. J., \& Carneiro, P. C. S. (2004). Modelos biométricos aplicados ao melhoramento genético (3rd ed.). Viçosa: UFV.

Fadel, A. L., Stuchi, E. S., Couto, H. T. Z., Ramos, Y. C., \& Mourão Filho, F. A. A. (2018). Trifoliate hybrids as alternative rootstocks for Valencia sweet orange under rainfed conditions. Scientia Horticulturae, 235, 397-406. https://doi.org/10.1016/j.scienta.2018.01.051

Fadel, A. L., Stuchi, E. S., Silva, S. R., Parolin, L. G., Oliveira, C. R., Muller, G. W., \& Donadio, L. C. (2019). Compatibility and horticultural performance of Pera sweet orange clones grafted to Swingle citrumelo rootstock. Bragantia, 78(4), 564-572. https://doi.org/10.1590/1678-4499.20190116

Fares, A., Bayabil, H. K., Zekri, M., Mattos Junior, D., \& Awal, R. (2017). Potential climate change impacts on citrus water requirement across major producing areas in the world. Journal of Water and Climate Change, 8, 576-591. https://doi.org/10.2166/wcc.2017.182

França, N. O., Amorim, M. S., Girardi, E. A., Passos, O. S., \& Soares Filho, W. S. (2016). Performance of Tuxpan Valencia sweet orange grafted onto 14 rootstocks in northern Bahia, Brazil. Revista Brasileira de Fruticultura, 38(4), 1-9. http://doi.org/10.1590/0100-29452016684

Fundecitrus (Fundo de Defesa da Citricultura). (2018). Tree inventory and orange crop forecast for São Paulo and West-Southwest Minas Gerais citrus belt 2018/2019 (p. 144). Araraquara: Fundo de Defesa da Citricultura. Retrieved November 12, 2019, from http://www.fundecitrus.com.br/pdf/pes_relatorios/Tree_ Inventory_and_Orange_Crop_Forecast_2018-2019.pdf

Fundecitrus (Fundo de Defesa da Citricultura). (2019). Tree inventory and orange crop forecast for São Paulo and West-Southwest Minas Gerais citrus belt 2019/2020 (p. 136). Fundo de Defesa da Citricultura: Araraquara. Retrieved January 14, 2020, from http://www.fundecitrus.com.br/pdf/pes_relatorios/2019_05_ 24_Tree_Inventory_and_Orange_Crop_Forecast_2019-2020.pdf

Girardi, E. A., Cerqueira, T. S., Cantuarias-Avilés, T. E., Silva, S. R., \& Stuchi, E. S. (2017). Sunki mandarin and Swingle citrumelo as rootstocks for rain-fed cultivation of late-season sweet orange selections in northern São Paulo state, Brazil. Bragantia, 76(4), 501-511. http://doi.org/10.1590/1678-4499.2016.350

Köeppen, W. P., \& Geiger, R. (1936). Das geographische system der klimate. In W. Köppen \& R. Geiger (Eds.), Handbuch der klimatologie. Berlin: Borntrager. 
Legua, P., Bellver, R., Forner, J. B., \& Forner-Giner, M. A. (2011). Trifoliata hybrids rootstocks for Lane Late navel orange in Spain. Scientia Agricola, 68, 548-553. http://doi.org/10.1590/S0103-90162011000500006

Mantel, N. (1967). The detection of disease clustering and generalized regression approach. Cancer Research, 27(2), 209-220. Retrieved May 21, 2020, from https://cancerres.aacrjournals.org/content/27/2_Part_1/ 209.full-text.pdf

Mingotti, S. A. (2005). Análise de dados através de métodos de estatística multivariada: Uma abordagem aplicada. Belo Horizonte: UFMG.

Nonino, E. A. (1995). Variedades de laranjas para fabricação de sucos. Laranja, 16(1), 119-132.

Passos, O. S., Souza, J. S., Bastos, D. C., Girardi, E. A., Gurgel, F. L., Garcia, M. V. B., ... Soares Filho, W. S. (2018). Citrus industry in Brazil with emphasis on tropical areas. In M. Sajid (Ed.), Citrus-health benefits and production technology (pp. 1-21). London: IntechOpen. https://doi.org/10.5772/intechopen.80213

Pearce, S. C., \& Dobersek-Urbanc, S. (1967). The measurements of irregularity in growth and cropping. Horticultural Science, 42, 295-305. https://doi.org/10.1080/00221589.1967.11514216

Pompeu Junior, J. (2005). Porta-enxertos. In D. Mattos Júnior, J. D. De Negri, R. M. Pio, \& J. Pompeu Junior (Eds.), Citros (pp. 61-104). Campinas: Instituto Agronômico: Fundag.

Pompeu Junior, J., \& Blumer, S. (2009). Híbridos de trifoliata como porta-enxertos para a laranjeira Valência. Pesquisa Agropecuária Brasileira, 44(7), 701-705. https://doi.org/10.1590/S0100-204X2009000700008

Pompeu Junior, J., \& Blumer, S. (2011). Citrandarins e outros híbridos de trifoliata como porta-enxertos para laranjeira Valência. Citrus Research \& Technology, 32, 133-138. https://doi.org/10.5935/2236-3122. 20110016

Pompeu Junior, J., Laranjeira, F. F., \& Blumer, S. (2002). Laranjeira Valência enxertadas em híbridos de trifoliata. Scientia Agricola, 59, 93-97. http://doi.org/10.1590/S0103-90162002000100014

Ramos, Y. C., Stuchi, E. S., Girardi, E. A., Leão, H., Gesteira, A. S., Passos, O. S., \& Soares Filho, W. S. (2015) Dwarfing rootstocks for Valencia sweet orange. Acta Horticulturae, 1065, 351-354. https://doi.org/ 10.17660/ActaHortic.2015.1065.42

Ribeiro, R. V., Espinoza-Núñes, E., Pompeu Junior, J., Mourão Filho, F. A. A., \& Machado, E. C. (2014). Citrus rootstocks for improving the horticultural performance and physiological responses under constraining environments. In P. Ahmad, M. R. Wani, M. M. Azooz, \& L. S. T. Tran (Eds.), Improvement of Crops in the Era of Climatic Changes (pp. 1-37). Springer, New York Heidelberg Dordrecht London. https://doi.org/ 10.1007/978-1-4614-8830-9_1

Rodrigues, A. S., Barbosa, C. J., Soares Filho, W. S., \& Freitas-Astúa, J. (2014). Comportamento de híbridos de citros em relação à infecção natural pelo Citrus tristeza vírus e à presença de sintomas de descamamento eruptivo. Revista Brasileira de Fruticultura, 36(3), 731-737. https://doi.org/10.1590/0100-2945-179/13

Rolim, G. S., Sentelhas, P. C., \& Barbieri, V. (1998). Planilhas no ambiente Excel para os cálculos de balanços hídricos: normal, sequencial, de cultura e de produtividade real e potencial. Revista Brasileira de Agrometeorologia, 6, 133-137. Retrieved June 21, 2020, from https://www.researchgate.net/publication/ 285636209

Schinor, E. H., Cristofani-Yaly, M., Bastianel, M., \& Machado, M. A. (2013). Sunki Mandarin vs Poncirus trifoliata hybrids as rootstocks for Pera sweet orange. Journal of Agricultural Science, 5, 190-200. http://doi.org/10.5539/jas.v5n6p190

Siebert, T., Krueger, R., Kahn, T., Bash, J., \& Vidalakis, G. (2010). Descriptions of new varieties recently distributed from the Citrus Clonal Protection Program. Citrograph, 1, 20-26. Retrieved June 21, 2020, from https://citrusvariety.ucr.edu/citrus/documents/Siebert_etal_2010_CCPP_New_Varieties_CitrographMarchA pril2010.pdf

Sneath, P. H., \& Sokal, R. R. (1973). Numerical taxonomy: The principles and practice of numerical classification. San Francisco: W.H. Freeman. http://doi.org/10.1086/408956

Sokal, R. R., \& Rohlf, F. J. (1962). The comparison of dendrograms by objective methods. Taxon, 11, 33-40. https:/doi.org/10.2307/1217208 
Spreen, T. H., Gao, Z., Fernandes Jr, W., \& Zansler, M. L. (2020). Global economics and marketing of citrus products. In M. Talon, M. Caruso, \& F. G. Gmitter Jr (Ed.), The Genus Citrus (pp. 471-493). Duxford: Woodhead Publishing. https://doi.org/10.1016/B978-0-12-812163-4.00023-1

Yildiz, E., Demirkeser, T. H., \& Kaplankiran, M. (2013). Growth, yield, and fruit quality of Rhode Red Valencia and Valencia Late sweet oranges grown on three rootstocks in eastern Mediterranean. Chilean Journal of Agricultural Research, 73, 142-146. http://doi.org/10.4067/S0718-58392013000200009

Zekri, M. (2000). Citrus rootstocks affect scion nutrition, fruit quality, growth, yield and economical return. Fruits, 55(4), 231-239. https://doi.org/10.21273/HORTSCI.35.3.499C

\section{Copyrights}

Copyright for this article is retained by the author(s), with first publication rights granted to the journal.

This is an open-access article distributed under the terms and conditions of the Creative Commons Attribution license (http://creativecommons.org/licenses/by/4.0/). 